\title{
Perspective
}

PERSPECTIVE Actualité en histoire de l'art

2| 2009

L'Espagne

\section{Art wisigoth en Hispania : en quête d'une révision nécessaire}

Visigoth Art in Hispania: in Search of a Much-Needed Reevaluation

Die westgotische Kunst in Hispania: auf der Suche nach einer notwendigen

Überprüfung

Arte visigota in Hispania: in cerca di una revisione necessaria

Arte visigodo en Hispania: en busca de una necesaria revisión

\section{Gisela Ripoll et Eduardo Carrero}

\section{(2) OpenEdition}

Journals

Édition électronique

URL : http://journals.openedition.org/perspective/1381

DOI : $10.4000 /$ perspective. 1381

ISSN : 2269-7721

Éditeur

Institut national d'histoire de l'art

Édition imprimée

Date de publication : 30 juin 2009

Pagination : 256-276

ISSN : $1777-7852$

Référence électronique

Gisela Ripoll et Eduardo Carrero, "Art wisigoth en Hispania : en quête d'une révision nécessaire », Perspective [En ligne], 2 | 2009, mis en ligne le 24 juillet 2014, consulté le 01 octobre 2020. URL : http:// journals.openedition.org/perspective/1381; DOI : https://doi.org/10.4000/perspective.1381 


\title{
Art wisigoth en Hispania: en quête d'une révision nécessaire
}

\author{
Gisela Ripoll et Eduardo Carrero
}

Les Wisigoths ont presque toujours été vus sous deux angles : d'un côté, comme l'un des peuples barbares ayant envahi l'Occident et responsables de la chute de l'Empire romain ; de l'autre, comme les artisans de la cohésion politique, territoriale et religieuse en Hispania, devenus, pour cette raison, l'instrument politique de la droite la plus opiniâtre dans les années 1940, lorsque le " germanisme " fut mis au service d'une construction historiciste du nationalisme espagnol. On constate aisément que le franquisme ne fit montre d'aucune originalité : la justification historique d'un pouvoir politique par la monarchie wisigothe fut une constante tout au long du Moyen Âge. Beaucoup de gouverneurs prirent comme référence l'unité territoriale wisigothe brisée par l'invasion musulmane, la mémoire des rois du IV au VIII ${ }^{\mathrm{e}}$ siècle finissant par être aussi célébrée, voire plus, que celle des contemporains eux-mêmes.

L'étude de l'art et de la culture wisigothiques est par conséquent difficile et délicate, mais la recherche scientifique menée ces dernières années par différents groupes de chercheurs - historiens ainsi qu'archéologues, philologues et historiens de l'art - permet néanmoins une lecture renouvelée du processus de migration, de l'installation des Wisigoths dans le sud de la Gallia, de leur passage en Hispania et de la construction d'un nouveau royaume, préparant ainsi les bases pour l'édification de l'Europe médiévale. Les Wisigoths et leurs manifestations culturelles et artistiques sont aujourd'hui perçus comme faisant partie d'une période d'innovation et de transformation, l'Antiquité tardive, qui se déroule de la fin du monde romain au début du Moyen Âge. Comprendre comment on en est arrivé à une définition de ce que l'on nomme wisigothique permet également de cerner les problèmes auxquels est confrontée la communauté scientifique dans l'identification de cet art.

\section{La naissance d'une période historique distincte}

Avec le XVII ${ }^{\mathrm{e}}$ siècle et la découverte des catacombes chrétiennes de Rome par Antonio Bosio, une nouvelle archéologie est née, l'archéologie paléochrétienne, qui s'est concrétisée aux XIX ${ }^{e}$ siècle grâce aux travaux de Giovanni Battista de Rossi (1822-1894), dans les recherches de Joseph Wilpert (1857-1944) et, par la suite, dans la création à Rome du Pontificio Istituto di Archeologia Cristiana par Pie XI en 1925. Si, l'intérêt s'est d'abord porté sur les premières manifestations d'inhumations chrétiennes, essentiellement les catacombes et les sarcophages,

Gisela Ripoll est professeur d'archéologie à l'université de Barcelone depuis 1992. Son enseignement et sa recherche se concentrent sur l'archéologie de l'Antiquité tardive et sur les Wisigoths. Elle a notamment obtenu le Prix Raoul Duseigneur, décerné par l'Institut de France, et une bourse « senior " à l'Institute for Advanced Study de Princeton University.

Eduardo Carrero est maître de conférences à l'Universitat des Îles Baléares, à Majorque. Ses travaux portent sur la fonctionalité des édifices médiévaux, notamment les formules architectoniques permettant de répondre aux nécessités liturgiques. Parmi ses travaux se distinguent ceux sur la cathédrale d'Oviedo, sur l'importance de la liturgie dans l'apparition des églises doubles en Occident, et sur les palais épiscopaux de la péninsule ibérique. 
l'archéologie paléochrétienne s'est peu à peu concentrée sur l'étude des lieux de culte. Les édifices ecclésiastiques ont constitué les seuls témoignages de l'architecture monumentale et de son iconographie ; d'où les travaux fondateurs de Richard Krautheimer, de Ranuccio Bianchi Bandinelli, d'André Grabar ou de Friedrich Gerke, pour ne citer que les plus connus. Mais la naissance de l'histoire de l'art et de l'archéologie paléochrétiennes ne peut être dissociée d'un autre domaine fondamental, l'étude du Bas-Empire romain et de la fin du monde ancien. Depuis le XVIII ${ }^{\mathrm{e}}$ siècle, et surtout depuis Edward Gibbon, les historiens ont débattu - et débattent encore aujourd'hui - des causes de la fin de l'Empire romain et de sa datation. L'imposant History of the Decline and Fall of the Roman Empire de Gibbon et le Mahomet et Charlemagne d'Henri Pirenne furent les chevaux de bataille des chercheurs pendant presque tout le XXe siècle (GIBBON, 1776-1789; PIRENNE, 1937).

À toutes ces contributions et controverses s'ajoute en outre un nouveau concept, celui de Spätantike, l'Antiquité tardive, qui a fait l'objet de nombreuses révisions (MARTIN, 1976, en pleine effervescence de cette nouvelle discipline ; le status quaestionis de GIARDINA, 1999 ; la vision anglo-saxonne récente avec JAMES, 2008 ; pour l'Hispania, l'état des lieux selon ARCE, 2005a). Au tout début du XXe siècle, l'un des grands historiens de l'art du moment, Alois Riegl, publie Spätrömische Kunstindustrie (RIEGL, 1901-1923). Il s'agit de l'une des premières tentatives de formalisme esthétique et l'une des premières analyses artistiques sous l'angle du petit matériel archéologique. Ce que l'on nomme " art mineur » y est mis définitivement au même niveau que l' " art majeur " et l'on commence à prendre en considération l'existence dans le monde romain d'une période " tardive " ayant ses spécificités (RIPOLL, RIPOLL, 1988 ; SCARROCCHIA, 1995 ; CAILLET, 2001). C'est Henri-Irenée Marrou, dans son immense travail en tant qu'historien des textes mais aussi comme spécialiste de l'art et de la culture, qui réclame une fois pour toutes que l'on ne parle plus de la fin d'un monde ancien mais d'une nouvelle période dotée de caractéristiques propres : l'Antiquité tardive (MARROU, 1938, 1977, 1978 ; voir aussi PALANQUe, 1978 ; HILAIRe, 1999 ; MOlina GómeZ, 2002 ; Riché, 2003). Période, ou plutôt civilisation, à laquelle il a attribué un caractère double : " elle [l'Antiquité tardive] ne se laisse pas réduire à l'unité, mais se développe selon deux lignes : d'un côté l'Empire qu'il faut maintenir et sauver malgré la pression des barbares [...], de l'autre la religion personnelle fondée sur la foi, celle des derniers païens, celle surtout du Christianisme triomphant [...]" (MARROU, 1968, p. 392).

L'ensemble de ces études, brièvement évoquées ici, a indubitablement marqué les travaux menés en Espagne par deux grands maîtres : Pere de Palol et Helmut Schlunk, dont il faut souligner l'influence décisive dans le catalogage et l'inventaire des monuments dits paléochrétiens de la péninsule ibérique (PALOL, 1967, [1940] 1991 ; SCHLUNK, 1947 ; SCHLUNK, HAUSCHILD, 1978), qui couvrent une chronologie allant du IV siècle, moment de l'implantation du christianisme, au VI e siècle, considéré comme un tournant dans l'architecture et la politique, du fait de l'arrivée des Wisigoths en Hispania. C'est précisément l'apparition des peuples barbares en Occident qui a conduit les chercheurs à envisager une rupture par rapport aux vestiges archéologiques et monumentaux de la période chrétienne (BARATTE et al., 2008). Pour cette raison, Palol a divisé, non sans perspicacité et habileté, la période politique du royaume hispanique des Wisigoths en deux grandes étapes. La première, dite époque wisigothique, se déroule entre le début de l'implantation des Wisigoths dans la péninsule en 507, après leur défaite contre les Francs à Vouillé (Vogladum), où Alaric II trouva la mort, et 589, année de la célébration du III ${ }^{e}$ Concile de Tolède et de la conversion de la gens gothorum au catholicisme. L'année 589 marque en effet la fin des différenciations 
1. Carte montrant I'implantation des centres administratifs de I'Église en Hispania, avant et après 589, année du III Concile de Tolède [PALOL, 1967, mapa V].
2. Frontispice d'Enrique Flórez, España Sagrada, XVIII, Madrid, 1754.

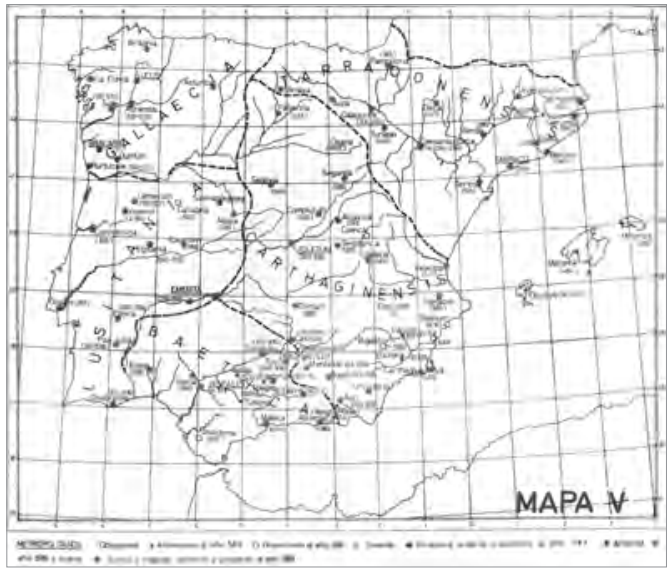

entre Wisigoths et Romains et ainsi l' " unification " religieuse, culturelle et territoriale des peuples vivant dans l'ancienne Hispania (fig. 1). Pour Palol, la seconde étape de l'art et de l'archéologie wisigothiques est celle époque hispano-wisigothique, et qui court de 589 à 711 , cette dernière date, celle de l'arrivée des Arabes, étant couramment perçue comme la clé pour comprendre la fin du monde ancien et le début d'une nouvelle ère. Pour l'historiographie espagnole, l'année 711 est presque aussi importante dans l'imaginaire collectif que le changement de millénaire en l'an Mil, ou 1492, avec l'expulsion des juifs d'Espagne et la découverte de l'Amérique, pour citer deux exemples légendaires.

Si la recherche espagnole a suivi de près les différents chemins que prenait le débat en France, en Italie et en Allemagne, l'école anglo-saxonne a été peu entendue. Cependant, l'ouvrage fondamental d'Arnold Hugh Martin Jones, The Later Roman Empire, qui a marqué nettement un avant et un après dans la connaissance de l'Antiquité tardive du point de vue de l'économie, de la société et de l'administration, bien qu'elle omette presque intentionnellement l'archéologie, est encore aujourd'hui un point de départ obligé pour toute personne qui s'intéresse à cette période (JONES, 1964). Peter Brown a ensuite introduit un des aspects essentiels de cette période, à savoir l'aspect religieux (BROWN, 1971, 1978, 1982, 1996 ; BROWN et al., 1997 ; HOWARD-JOHNSTON, HAYWARD, 1999), sujet que Jones, loin de la tradition confessionnelle et séculaire qui a caractérisé l'école française, avec Marrou à sa tête, n'avait pas abordé. Aussi bien Jones que Brown sont devenus les "pères fondateurs " d'une grande école anglo-saxonne (CAMERON, 1993 ; LIEBESCHUETZ, 2001 ; WARD-PERKINS, 2005 ; WiCKHAM, 2005). En France la seconde moitié du XXe siècle a été marquée par une triade scientifique, composée de Paul-Albert Février, Charles Pietri et Noël Duval, qui a influencé toutes les études postérieures. Collègues et amis, ils dirigèrent ensemble de grands projets parmi lesquels se distinguent la Topographie chrétienne des cités de la Gaule des origines au milieu du VIII siècle et le XI Congrès international d'archéologie chrétienne, Lyon, Vienne, Grenoble,

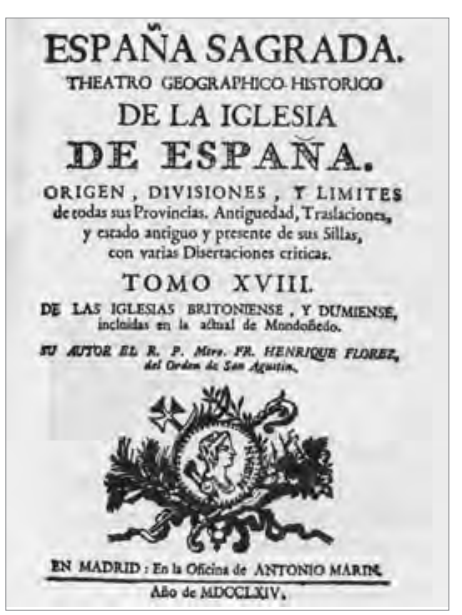
Genève et Aoste célébré en 1986 (voir l'hommage à Février et Pietri dans DUVAL, 1992). Le monde anglo-saxon, depuis Princeton, possède également sa triade, avec Peter Brown, Glen Bowersock et Oleg Grabar (BOWERSOCK, BROWN, GRABAR, 1999, 2001).

\section{Vers une définition de l'art wisigoth}

Définir ce qui est wisigoth n'est en rien propre au XX⿳⺈冂大 siècle ou aux courants académiques contemporains. Le souci de retracer l'histoire du royaume hispano-wisigothique et, ce qui est plus intéressant, d'établir des liens de continuité avec la période en question fut une préoccupation constante depuis le haut Moyen Âge. La simple présence 
de la Chronique d'Alphonse III des Asturies, qui attribue à son prédécesseur Alphonse II l'instauration à Oviedo d'une cour à l'image de celle des Wisigoths à Tolède, est déjà suffisamment éloquente (BANGO, 1985). On retrouve toute une kyrielle de traditions, de lieux de sépultures et de vestiges des rois wisigoths tout au long du Moyen Âge dans l'occident péninsulaire, même si aucun gouverneur ne s'est autant acharné qu'Alphonse X pour identifier les origines de la monarchie du XIII ${ }^{e}$ siècle et les lignes de continuité permettant de la faire remonter à celle qui précédait l'invasion musulmane (FERNÁNDEZ-ORDÓÑEZ, 2000 ; IZQUIERDO, 2001 ; ALONSO, 2005, 2007, 2008).

Au XVI siècle, Charles Quint et Philippe II commandèrent une Histoire de l'Espagne à plusieurs chroniqueurs (RIPOLL, 1989). Florián de Ocampo (1490-1558) conçut ce qui devint la Crónica General de España, qui tente de couvrir l'histoire espagnole de ses origines à Charles Quint. On lui doit les cinq premiers livres de la Crónica General de España: desde los orígenes hasta el siglo III a.C. (1544 et 1553), travail qui fut poursuivi par Ambrosio de Morales (1513-1591) avec la Crónica General de España: desde el siglo III a.C. hasta el año 1000 (1574). Morales est le premier à réellement prendre en considération les vestiges et le matériel archéologiques romains mais aussi arabes. Ses recherches historico-archéologiques à Alcalá de Henares sont remarquables et lui ouvrirent la voie pour l'étude des reliques chrétiennes dont textes et archéologie témoignent, notamment dans Las antigüedades de las ciudades de España (1573).

Il faut attendre le XVIII ${ }^{\mathrm{e}}$ siècle pour enregistrer une nouvelle phase importante dans la reconstruction historique hispanique. La monumentale España Sagrada d'Enrique Flórez ${ }^{1}$ (1702-1773 ; fig. 2) est, aujourd'hui encore, le point de départ pour toute recherche (RIPOLL, 1989). L'élaboration de cette histoire en plusieurs volumes s'appuie sur une connaissance directe des antiquités : Flórez parcourut le territoire espagnol dès 1742 afin de rassembler du matériel numismatique et épigraphique, et d'étudier les monuments majeurs. Il constitua en outre la première liste des rois goths, dont se souviennent si bien les Espagnols scolarisés sous le franquisme qui les apprenaient par cœur, des premiers (Athaulf, Sigeric, Wallia) aux derniers (Égika, Witiza, Rodrigue), en passant par les plus glorieux (Leovigild, Recarède). La fin du XVIII ${ }^{e}$ siècle fut marquée par la mise au jour de Segóbriga, l'un des sites emblématiques du monde romain péninsulaire doté d'une église tardive, que fit connaître Joseph Córnide dans Noticias de las antigüedades de Cabeza de Griego (1799). Cette découverte s'ajouta à la compilation d'épigraphie chrétienne réunie par Juan Francisco Masdeu dans son Historia Crítica de España en vingt volumes (vol. IX dédié aux inscriptions chrétiennes, 1791).

Ce n'est qu'à la fin du XIX ${ }^{e}$ siècle qu'une autre grande découverte vint mettre de nouveau l'archéologie et l'art wisigoth sur le devant de la scène. Il s'agit du trésor de Guarrazar (Huerta de Guarrazar, Tolède), révélé en 1858 (CAILLET, 1985, p. 218-227 ; BALSAMEDA, 1995, 1996 ; PEREA, 2001 ; RIPOLL, 2004 ; fig. 3). Les couronnes et les croix retrouvées furent remarquées et achetées par un
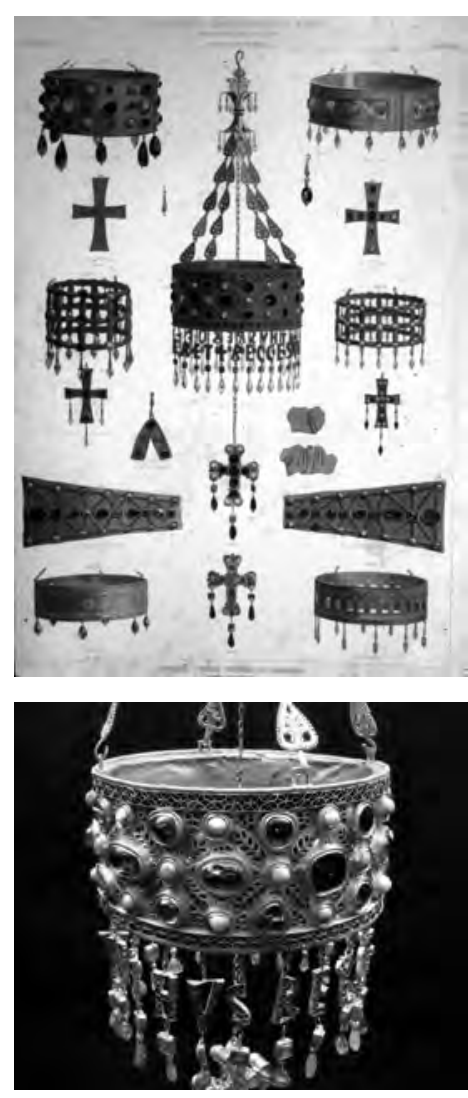

3. Le trésor de Guarrazar : a. planche tirée de Monumentos Arquitectónicos de España, Madrid, 1879 ; b. couronne votive offerte par le roi Receswinth, VII siècle, Madrid, Museo Arqueológico Nacional. 
officier français à la retraite résidant en Espagne, Adolphe Herouart. Les quatorze couronnes furent vendues et fondues par des orfèvres à qui Herouart avait montré le lot, mais de nouvelles fouilles mirent au jour huit autres couronnes votives et six croix en or serties de pierres précieuses, destinées à être suspendues. Un an plus tard, le Musée de Cluny à Paris fit l'acquisition de ces pièces. L'apprenant, la Real Academia de la Historia envoya son académicien José Amador de los Ríos faire des fouilles supplémentaires (Ríos, 1861), qui débouchèrent sur la découverte de quatre nouvelles couronnes. Malgré le courroux espagnol, une couronne se retrouva au Musée de Cluny, les trois autres à la Real Armería du Palacio Real de Madrid (dont deux furent volées en 1921 et ne furent jamais retrouvées). Des années plus tard, en 1940, les gouvernements de Franco et de Pétain s'accordèrent pour établir un échange de biens culturels. Les couronnes du trésor de Guarrazar ainsi que la Dame d'Elche retournèrent en Espagne au Museo del Prado, mais trois couronnes demeurèrent en France et le reste finit par être intégré aux collections du Museo Arqueológico Nacional de Madrid, à partir de 1943. Si un objet artistique a un jour été utilisé comme instrument politique, c'est bien le cas du trésor de Guarrazar, en particulier la couronne de Receswinthe (VII siècle, Madrid, Museo Arqueológico Nacional), symbole d'une Espagne unifiée et catholique telle que l'envisageait le franquisme.

À partir de la seconde moitié et surtout de la fin du XIX siècle commence ce que l'on pourrait appeler la "glorification " du passé romain et wisigothique, qui dura pratiquement jusqu'à la moitié du Xx $x^{\text {e }}$ siècle. Parallèlement, une archéologie de type monumental, fondée sur la récupération des édifices en tant que document historico-artistique, a commencé à se développer. Pour ce faire, une série de publications fut créée à la demande de la Real Academia de Bellas Artes de San Fernando, sous le nom de Monumentos Arquitectónicos de España (18591881), dont l'objectif était de faire connaître les principaux monuments architecturaux espagnols, ainsi que certains objets d'art ou éléments décoratifs. La sélection des édifices est arbitraire, de même que leur place dans les différents volumes. À cette œuvre magistrale ont participé, entre autres, José Amador de los Ríos, Rodrigo Amador de los Ríos, Manuel de Assas, Pedro de Madrazo et Aureliano Fernández Guerra, accompagnés de dessinateurs hors pair. On trouve chez Aureliano Fernández Guerra un intérêt manifeste pour l'archéologie chrétienne, l'iconographie funéraire des sarcophages (en particulier ceux de Layos et celui de Hellín) et l'épigraphie (FERNÁNDEZ GUERRA, 1866). Son œuvre est particulièrement intéressante

4. Site Internet du Corpus Inscriptionum Latinarum (CIL II) (www2. uah.es/imagines_cilii/Inscriptiones_MAN/ Lusitania Mapa.htm).

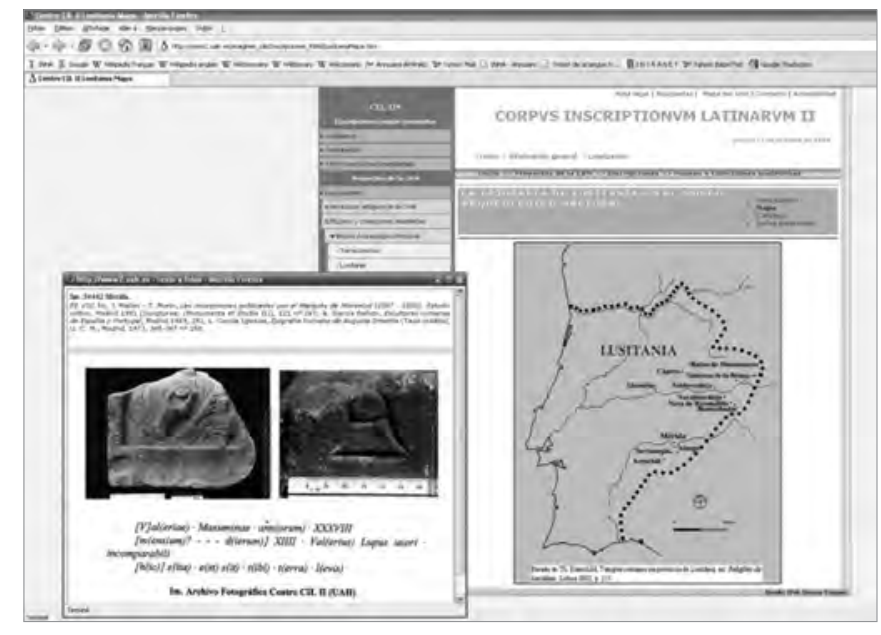
puisqu'elle établit une des premières périodisations pour l'art de cette période : pré-Constantin, époque de Constantin, domination wisigothique et reconquête (MAIER ALLENDE, 2006).

Concernant l'épigraphie et la fin du XIX siècle, on ne peut manquer de rappeler l'une des contributions les plus importantes, celle d'Emil Hübner et de son imposant Inscriptiones Hispaniae Christianae (1871), intégré par la suite au Corpus 
Inscriptionum Latinarum, CIL II ${ }^{2}$ (fig. 4), qui instaure une nouvelle méthodologie de travail et l'obligation de prendre en compte l'ensemble des sources archéologiques et textuelles dans toute tentative de reconstruction historique ou historico-artistique de la fin du monde romain péninsulaire.

Dans cette même optique, bien que centré sur l'architecture, José Caveda y Nava avança l'une des propositions les plus novatrices mais, en même temps, la moins diffusée de toutes celles proposées par une historiographie de l'architecture de l'Antiquité tardive péninsulaire encore balbutiante. L'érudit et poète asturien suggéra la possibilité d'une continuité culturelle depuis le Bas-Empire en qualifiant les bâtiments d' « architecture latine dans la monarchie gothe " (CAVEDA Y NAVA, 1848). Sa proposition semble avoir un certain fondement. Après avoir réalisé une synthèse des sources épigraphiques et autre documentation récente relative aux édifices et constructions chrétiennes hispano-wisigothiques, Caveda parvint à la conclusion qu'aucun des monuments conservés dans la péninsule ne pouvait dater de l'époque hispano-wisigothique : ils ne pouvaient être antérieurs au $\mathrm{IX}^{\mathrm{e}}$ siècle puisqu'aucun élément n'est suffisamment " classique » - si ce n'est ce qui serait ensuite qualifié de préroman asturien - pour être considéré comme un ultime soubresaut de la fin du monde classique. De toute évidence, par la chronologie ainsi établie comme dans ses propositions théoriques, Caveda fut en avance sur son temps en dessinant, sans le savoir, les deux axes majeurs de l'historiographie la plus récente : d'une part la remise en question chronologique des édifices conservés et, d'autre part, le fait que l'architecture du royaume des Asturies soit véritablement représentative de la fin de l'Antiquité tardive.

Dans sa systématisation théorique de l'architecture médiévale péninsulaire, Vicente Lampérez y Romea montra peu d'intérêt pour le monde antérieur au XIII ${ }^{e}$ siècle, au-delà d'une approche uniquement descriptive et d'une classification formelle relevant du positivisme inhérent aux écoles de recherche ou de restauration inspirées par les doctrines d'Eugène Viollet-le-Duc (LAMPÉREZ Y ROMEA, [1908-1909] 1999). Au contraire, au sud de la péninsule, le goût prononcé de Manuel Gómez-Moreno pour le monde wisigoth fut certainement lié à sa fréquentation, dès son plus jeune âge, d'Emil Hübner, alors que celui-ci s'occupait de réunir le matériel pour l'élaboration du Supplementum, dans le volume dédié à l'Hispania du Corpus Inscriptionum Latinarum, le CIL II (STYLOW, GIMENo, 2004). Cette relation intellectuelle fut à l'origine des Monumentos romanos y visigóticos de Granada, publiés en 1889 (GÓMEZ-MORENo, [1889] 1988). Toutefois, ce n'est pas avant le début du XX $X^{e}$ siècle, en plein travail sur le Catálogo Monumental de España, pour lequel il se chargea des provinces d'Ávila et de Salamanque (1901-1902), de León (1903) et de Zamora (1906), qu'il effectua la redécouverte virtuelle de l'église de San Pedro de la Nave située dans cette dernière, en utilisant l'épigraphie comme élément de datation architecturale pour la situer entre le VII et le VIII ${ }^{\mathrm{e}}$ siècle (GÓMEZ-MORENO, 1906 ; GIMENO, 2004).

De son côté, en Catalogne, l'historien et architecte Puig i Cadafalch consacra l'essentiel de ses recherches à la période comprise entre le $\mathrm{X}^{\mathrm{e}}$ et le XII ${ }^{\mathrm{e}}$ siècle, et en particulier aux origines de l'art roman en tant que prémisses de la culture catalane. Il reconnut cependant la singularité de la basilique tarragonaise de San Fructuoso (PUIG I CADAFALCH, 1936a), et sa contribution fut fondamentale pour la compréhension et la connaissance des églises de Terrassa, ensemble remarquable dont il fut le restaurateur (PUIG I CADAFALCH, 1936b, 1948 ; fig. 5). Son interprétation a prévalu jusqu'aux nouveaux apports des récentes recherches archéologiques (GARCiA, Moro, TUSET, 2009). Son ouvre posthume (PUIG I CADAFALCH, 1961), qui réunit des conférences données lors d'un cycle à l'Institut d'Histoire de l'Art et d'Archéologie de l'Université de Paris entre 1937 et 1940, est bien plus qu'un simple état des lieux. 
5. Vues axonométriques de l'église San Miquel du siège épiscopal de Terrassa, interprétée et restituée par Puig i Cadafalch comme un baptistère ; avant et après restauration [PUIG I CADAFALCH, 1948, fig. 8-9].

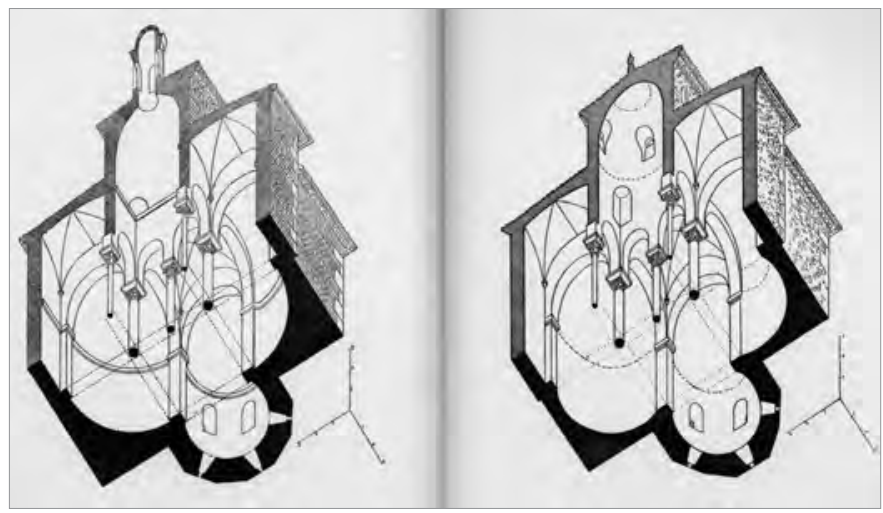

En 1940 voyait le jour le troisième volume de l'Historia de España, sous la direction de Ramón Menéndez Pidal, consacré au monde wisigoth. Emilio Camps Cazorla fut chargé de réaliser le chapitre sur les arts (CAMPS, 1940), même si, vingt ans plus tard, la continuelle révision de l'œuvre et les nouvelles publications et rectifications scientifiques - que l'on doit essentiellement à Helmut Schlunk et à Pere de Palol -, réclamèrent d'y joindre un addenda (LóPEZ SERRANO, 1963). Dans les années 1990 déjà, dans le nouveau volume qui complétait le travail historique des années 1940, le responsable du chapitre sur les arts était Pere de Palol lui-même (PALOL, [1940] 1991 ; exposé synthétique de la période dans OLMO, 1991).

En 1947, Helmut Schlunk signait les textes sur l'art wisigoth et asturien dans le volume consacré à l'Antiquité de la collection Ars Hispaniae. Depuis 1943, il était chargé de fonder le Deutsches Archaologisches Institut (DAI), Abteilung Madrid, et, surtout, il fut à l'origine d'un contrôle systématique de la rigueur scientifique des recherches sur les arts hispaniques entre le IV ${ }^{e}$ et le IX $\mathrm{X}^{\mathrm{e}}$ siècle. Au-delà de ses propositions pertinentes sur l'architecture, Schlunk fut le premier à traiter un problème fondamental du monde wisigoth : l'absence de miniatures, phénomène que l'on peut étendre au monde de la monarchie asturienne, en particulier à son "âge d'or" au IX" siècle (SCHLUNK, 1945). L'œuvre de Schlunk a été continué par d'autres chercheurs en attirant l'attention sur la continuité iconographique et l'héritage assumé par les œuvres du $\mathrm{X}^{\mathrm{e}}$ siècle, au moins pour les systèmes représentatifs et les travaux iconographiques, et sur les problèmes de datation pour les ermitages et les groupes monastiques rupestres de La Rioja (ÍÑIGUEZ, 1953 ; CID, 1980 ; FERNÁNDEZ SOMOZA, 2004).

Avec Schlunk, Palol fut l'autre rénovateur de la recherche en histoire de l'art et en archéologie paléochrétienne et wisigothique (RIPOLL, 1983 ; MOLIST, GURT, PALOL, 2002 ; GURT, GUARDIA, RIPOLL, 2005). Pour lui, l'époque wisigothique, de 507 à 589, est caractérisée par la production de parures et d'objets personnels trouvés dans les nécropoles de la Meseta, et la seconde époque, celle nommée hispano-wisigothique, comprise entre 589 et 711, par le développement d'une architecture monumentale. La différence - ou ce qu'il aimait appeler le " divorce " - est très claire à ses yeux entre les arts mineurs du travail du métal et la sculpture et l'architecture monumentales. Pour autant, Palol s'est consacré à étudier conjointement les deux manifestations et les deux étapes, considérant que les arts mineurs sont le fruit de l'innovation et la sculpture et l'architecture celui de la continuité. Son postulat de départ était qu'il n'y avait aucune rupture entre Antiquité tardive et le monde médiéval, mais bel et bien une continuité. Palol structura ses recherches autour de trois facteurs, pour lui évidents : l'adoption par les Wisigoths des traditions hispano-romaines et latino-méditerranéennes; la date clé de la célébration du III $^{\mathrm{e}}$ Concile de Tolède en 589 , symbole d'un " avant " purement wisigothique et d'un "après " hispano-wisigothique ; et enfin les réminiscences du wisigothique dans le nord-est de la péninsule au-delà de la date mythique de 711 . 
Avec Schlunk et Palol est admise, en histoire de l'art comme en archéologie, la confluence de trois cultures : germanique, romaine et byzantine, l'étape suivant le Concile de Tolède étant perçue comme une période d'intégration unique entre Wisigoths et Hispano-Romains. Les deux chercheurs, parfaits connaisseurs du monde romain, défendirent l'idée d'une période unique, introduisant au sein de l'historiographie espagnole le concept allemand de Spätantike au détriment de la traduction espagnole Antigüedad tardía [Antiquité tardive]. Ils établirent en outre des distinctions claires entre la période du IV au $\mathrm{VI}^{\mathrm{e}}$ siècle, l'époque wisigothique postérieure du début du VI $\mathrm{I}^{\mathrm{e}}$ siècle jusqu'à l'arrivée des Arabes, et le haut Moyen Âge. Les voies ouvertes par Palol et Schlunk ont été approfondies par de nouvelles générations de chercheurs qui ont profité de leur formation et de l'arrivée de la démocratie qui a entrainé une nouvelle étape pour l'archéologie et s'est implantée peu à peu dans les différentes communautés autonomes. Dans les années 1970, Jacques Fontaine publia les deux volumes de son Art préroman hispanique, une contribution importante car, en utilisant l'étiquette de préroman, elle traçait une ligne continue entre l'art paléochrétien et mozarabe, une unité culturelle à envisager au moins du $\mathrm{V}^{\mathrm{e}}$ au $\mathrm{X}^{\mathrm{e}}$ siècle (FONTAINE, 1973, 1977).

On ne peut clore ces débats autour de la définition de l'art wisigoth sans distinguer Javier Arce, l'un des chercheurs qui a contribué à la discussion et à la connaissance historique en analysant de nombreuses sources, à la fois textuelles, artistiques, archéologiques, épigraphiques, iconographiques, etc. Son livre sur le IV siècle ouvrit de nouvelles perspectives, une nouvelle façon de voir la fin du monde ancien en Hispania (ARCE, 1982) ; depuis lors, cet historien et archéologue a revendiqué un point de vue plus vaste, incorporant tous les éléments historico-culturels, économiques, religieux et artistiques qui permettent de voir sous un angle nouveau l'Antiquité tardive hispanique (ARCE, 1982, 1988, 2005b). La recherche actuelle, héritière de cette riche historiographie, se veut interdisciplinaire, même si nous sommes encore loin d'une vision cohérente et unanimement plébiscitée de ce que fut la réalité historique et archéologique de l'Hispania des $\mathrm{VI}^{\mathrm{e}}$ et VII ${ }^{\mathrm{e}}$ siècles (voir ARCE, 2005a pour un état des lieux).

Les aspects historiographiques que nous venons de mentionner montrent comment, pratiquement depuis le XVI ${ }^{\mathrm{e}}$ siècle, les historiens, spécialistes de l'art, archéologues et architectes ont cherché des méthodes et des explications argumentées pour identifier les manifestations artistico-culturelles de la géographie hispanique du IV e au VIII ${ }^{\text {e }}$ siècle et, plus particulièrement, l'art wisigoth. Et pourtant, aujourd'hui encore, on n'est arrivé à aucune réponse unanimement acceptée par la communauté scientifique, et encore moins à sa diffusion auprès d'un public plus général. Le débat reste ouvert sur trois fronts. D'une part, ce que nos prédécesseurs ont appelé les arts mineurs, c'est-à-dire les parures et objets personnels trouvés au sein des sépultures des grands cimetières castillans, dont l'interprétation est étroitement liée à la question de l'implantation des Wisigoths dans la péninsule. D'autre part, le débat central sur l'identification d'une architecture wisigothique, dont les critères ont été remis en question ces quinze dernières années à propos des édifices traditionnellement considérés comme tels. Si l'architecture est objet de controverse, la sculpture monumentale sert, quant à elle, de pierre de touche pour réviser la chronologie des églises en Espagne. L'analyse détaillée des trois points doit être menée pour comprendre les éléments mêmes de la polémique et pour permettre au lecteur de se faire sa propre opinion de ce qui relève ou peut relever du monde wisigoth en Hispania au vu des données dont on dispose actuellement. 
L'archéologie funéraire, parures et objets personnels : l'implantation des Wisigoths en Hispania

Les parures et autres objets personnels qui se trouvaient dans les sépultures des cimetières wisigoths dispersés sur la Meseta, entre le Douro et le Tage, sont un des points clés pour déterminer les caractéristiques de l'art et de l'archéologie wisigothiques. L'étude et l'analyse de ces nécropoles renvoient à deux questions historiques : l'implantation des Wisigoths dans la péninsule ibérique et les critères d'identité de ce peuple, directement liés à son ethnogenèse (RIPOLL, 2000, 2001a, 2001b, 2006).

Les Consularia Caesaraugustana, anciennement Chronica Caesaraugustana, signalent par deux fois - en 494 et 497 - l'arrivée et l'installation des Wisigoths en Hispania. Les deux citations, "Goti in Hispanias ingressi sunt " (CARDELlE DE HARTMANN, 2001, 494; CC, 7 la : 22) et, peu après, "Gotthi intra Hispanias sedes acceperunt" (497; CC, 75a : 23), sont l'une des pièces majeures de la polémique sur l'implantation des Wisigoths en Hispania à la fin du V siècle (RIPOLL, 1991, 2006 ; KULIKOWSKI, 2004 ; ARCE, 2005b ; KOCH, 2006). Certains auteurs pensent qu'il s'agissait uniquement de présence militaire, d'autres de militaires accompagnés de civils - leurs propres familles -, comme c'était souvent le cas, par exemple, pour les soldats ostrogoths ou les Vandales.

Quant aux sources matérielles, deux paramètres sont fondamentaux : d'une part la caractérisation archéologique des établissements ou structures d'habitat, et de l'autre les lieux d'inhumation, puisqu'il existe en général une dichotomie entre le monde des vivants et le monde des morts.

Les fouilles de ces dernières années, dans les alentours de Madrid (BARRoso et al., 1993 ; VigIL-ESCALERA GUIRAdo, 2000, 2005 ; MORÍn DE PABlos, BARROSO, 2005), ont dévoilé un type d'habitat inhabituel : une cabane en bois, superposée sur d'anciennes structures identifiées grâce aux traces des poteaux, semblable à la cabane nordique dite Longhaus associée aux barbares (HAMEROW, 2002). On trouve également quelques uillae, reconverties en habitat ou nécropole, mais qui ne ressemblent en rien aux dénommées nécropoles wisigothiques de la Meseta (RIPOLL, ARCE, 2000 ; CHAVARRÍA, 2007).

Les grandes nécropoles en plein air, aux sépultures alignées de la Meseta, comme Castiltierra, Duratón, Madrona, Cacera de las Ranas, El Carpio de Tajo, pour citer les plus emblématiques, conservent des parures et objets personnels qui datent de la fin du ve à la fin $\mathrm{du} \mathrm{VI}^{\mathrm{e}}$ siècle, avec une plus forte occurrence dans la première moitié du VI ${ }^{\mathrm{e}}$ siècle, et qui sont le fruit d'une mode identifiée comme wisigothique par les chercheurs au début du XX siècle (ZEISS, 1934 ; voir aussi RIPOLL, 1991, 1998), bien qu'on observe aussi un clair mélange d'éléments wisigothiques et romains, c'est-à-dire de mariages mixtes (JiMÉNEZ GARNICA, 1985, 1999 ; RIPOLL, 1993-1994 ; fig. 6). Généralement, les objets de ce type sont perçus comme des "symboles d'identité ", c'est-à-dire comme des éléments d'auto-représentation et d'auto-affirmation de l'individu au sein du groupe social, surtout à cette époque où l'on constate une altération dans le système culturel et dans l'organisation interne du groupe lui-même (BINFORD, 1971).

Avant de présenter le débat actuel sur la définition de l'art wisigoth, il est nécessaire de rappeler que l'implantation wisigothique dans le sud de la Gallia, qui a commencé au début du $\mathrm{V}^{\mathrm{e}}$ siècle et s'est poursuivie pendant presque cent ans, n'a pas laissé des restes archéologiques analogues à ceux de la Meseta. Les parures et objets personnels semblables trouvés en Gallia correspondent à un $\mathrm{VI}^{\mathrm{e}}$ siècle bien avancé, alors que la Narbonensis faisait partie du royaume wisigothique de Tolède, jusqu'à sa chute en 719 aux mains des Maures. 
L'information qu'offrent les textes - et le silence des vestiges matériels - a suscité de nombreuses questions de la part des historiens et archéologues tout au long du XXe siècle. Aujourd'hui, l'école allemande, avec Völker Bierbrauer en tête (BIERBRAUER, 1994a, 1994b, 1999), se trouve sans réponse face à ce que ce dernier appelle " miracle archéologique " et ne parvient à expliquer pourquoi il n'existe aucune preuve matérielle qui témoigne de la présence de la population gothe de la Gallia du ve siècle, alors que sa présence est attestée en Hispania presque un siècle plus tard. Michel Kazanski pense que les Goths installés en Gallia peuvent être identifiés à partir de cinq peignes en os, qui rappellent l'ancienne culture goth-danubienne de Chernajov, découverts dans des uillae rurales (KAZANSKI, 1985 ; KAZANSKI, LAPART, 1995). L'hypothèse est intéressante mais la documentation archéologique et le lien direct de ces objets avec les résidences aristocratiques demeurent rares et ténus; de plus, elle ne résout pas le problème des établissements et des preuves matérielles présentes en Hispania.

À ce stade, il faut poser la question de la signification sociale et culturelle des nécropoles de la Meseta et aborder le débat des dernières années qui cherche à déterminer si les nécropoles wisigothiques sont vraiment le lieu de sépulture d'individus d'origine wisigothe. Pour cela, il est nécessaire de se tourner vers les études d'ethnogenèse qui ont pour objectif de définir et d'établir les critères qui constituent l'identité d'un individu, dans le cas présent, d'origine wisigothe. L'école de Vienne, avec à sa tête Herwig Wolfram et Walter Pohl, conclut, à l'examen d'auteurs comme Virgile, Tacite, Ammien Marcellin, Saint Augustin, Orose et Saint Isidore de Séville, qu'il existe des éléments qui, de façon globale et sans que l'existence de l'un implique celle de l'autre, permettent d'établir des différences ethniques ou des stratégies de distinction (WOLFRAM, 1990, 1994 ; POHL, 1997, 2000a, 2000b ; POHL, REIMITZ, 1998 ; GOETZ, JARNUT, POHL, 2003). Ainsi, on peut observer l'origo (l'origine), la lingua (la langue), la lex (la loi) et la religio (la religion). Mais quelle est la réalité archéologique ou artistique ? La réponse se trouve dans ces objets et accessoires vestimentaires retrouvés dans les cimetières de la Meseta.

Les chercheurs allemands, au cours des années 1940 et dans un contexte politique clair, identifièrent ces parures comme le « costume national goth " et le peuple wisigoth comme une ethnie ou race germanique complètement différente du reste de la population de la péninsule ibérique (GÖTZE, 1907 ; ÅBERG, 1922 ; ZEISS, 1934 ; REINHART, 1945 ; WERNER, 1942, 1946 ; MARTIN, 1991 ; BIERBRAUER, 1994a). Au milieu du $\mathrm{XX}^{\mathrm{e}}$ siècle, de nouvelles générations d'archéologues ont démontré la continuité du monde romain dans l'horizon wisigoth, en appréciant à sa juste mesure ce qu'on s'est mis à appeler « germanisme " face à une " romanité " forte (PALOL, 1956, [1940] 1991). Par la suite, la « Nouvelle Archéologie » en vint à nier toute possibilité de caractérisation d'un groupe ethnique à partir d'objets funéraires (LA ROCCA, 1989 ; KULIKOWSKI, 2004), allant ainsi à l'encontre de l'opinion d'autres chercheurs comme Lewis R. Binford
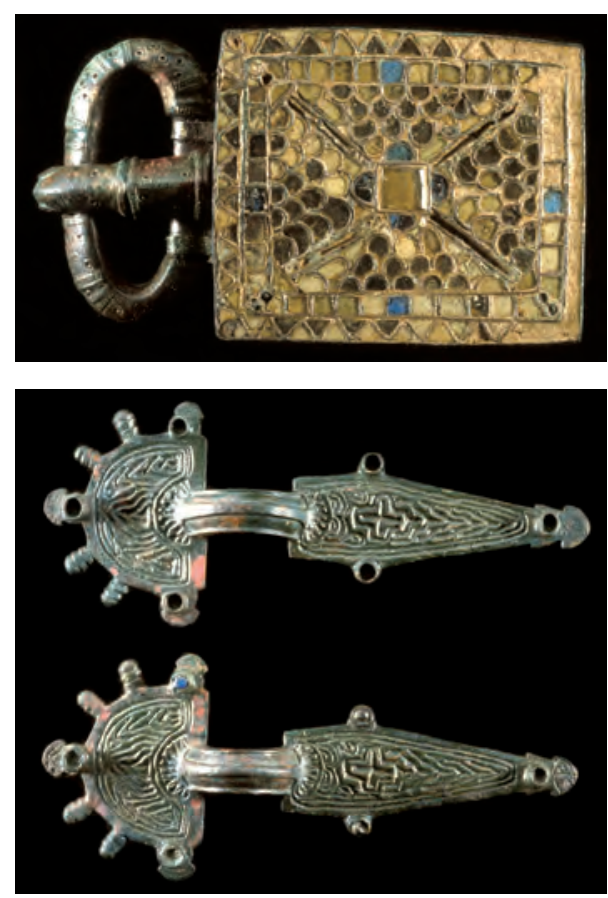
première moitié du $\mathrm{Vl}^{\mathrm{e}}$ siècle, Madrid, Museo Arqueológico Nacional : a. plaque-boucle de ceinture en cloisonné ;

b. fibules en bronze moulé. 
(BINFORD, 1971, 1983) qui continuent de considérer les pratiques funéraires comme un élément clé pour comprendre les sociétés du passé, la culture matérielle ayant une dimension symbolique qui affecte toutes les questions économiques et sociales d'une communauté (HODDER, 1986).Selon cette position méthodologique, la " Nouvelle Archéologie " affirme que ce que l'on considère comme les nécropoles wisigothiques de la Meseta, n'en sont pas (Collins, 2004). Mais si c'est le cas, la question se pose de savoir qui est enterré dans ces sépultures datées de la fin du $\mathrm{V}^{\mathrm{e}}$ à la fin du $\mathrm{VI}^{\mathrm{e}}$ siècle et découvertes presque exclusivement dans la Meseta. S'il ne s'agit ni de Vandales, ni d'Alains, ni de Suèves, ni de Goths, ni même de Grecs, de Syriens ou d'Africains, sont-ils par élimination romains ? Certains chercheurs considèrent qu'il s'agit de Romains gothisés (HEATHER, 1996), ce qui donne lieu à une autre série de questions : pourquoi accepter la possibilité d'une identification ethnique pour les Romains et non pour les Goths ? Pourquoi concevoir l'idée d'une mode gothe qui aurait été le fait des Romains et non des Goths ? Et pourquoi seulement des Romains de la Meseta? Pourquoi être enterré avec ces parures aurait-il été pour les Romains une forme d'émulation des Goths ? Pourquoi nier l'existence des Wisigoths en Hispania telle que l'indiquent les sources écrites? Une faille subsiste dans ce raisonnement.

Les parures et objets personnels " habillent " le mort, font partie de la vestimenta, la tenue funéraire du défunt, et sont des signes d'identité et d'auto-représentation qui constituent la vie symbolique d'un groupe social, utilisés généralement dans les moments où il est nécessaire de se réaffirmer socialement, culturellement et politiquement en tant que groupe (BINFORD, 1971 ; HÄRKE, 1992 ; RICHARDS, 1995 ; sur les Wisigoths plus particulièrement, RIPOLL, 1999, 2006). Les Goths de la Meseta, quel qu'en fût le motif, eurent besoin de réaffirmer leurs origines et leur status et utilisèrent pour cela les parures et objets personnels découverts dans les sépultures (LIEBESCHUETZ, 1998). Il est utile de rappeler ce que disait Ryszard Kapuściński au sujet de la culture en tant qu'identité dans un petit livre remarquable, Encountering the Other, dans lequel il affirme que la culture n'est pas immobile, statique et immuable, mais bien au contraire, dynamique, fluctuante et sujette aux changements et transformations provoqués par le contexte social et culturel, par l'environnement et par l'imaginaire même de chaque individu (KAPUŚCIŃSKI, 2005).

\section{Les études sur l'architecture et la sculpture : tradition et discordance}

Au Consejo Superior de Investigaciones Científicas (CSIC) de Madrid, les travaux de Luis Caballero sont ceux qui ont suscité le plus de polémiques ces dernières décennies. Centrée sur une lecture stratigraphique de l'architecture - qui inclut la sculpture comme une unité parmi d'autres -, la proposition du chercheur se fonde sur le retard chronologique de la plupart des églises jusque-là considérées comme wisigothiques et qui, à la suite de ses travaux archéologiques, ont été requalifiées comme datant de la "reconquête " - ou " repeuplement ", comme on dit actuellement - ou, en tout cas, construites après le VIII siècle. En débutant par Melque puis, par extension, en passant aux principaux exemples de l'architecture du haut Moyen Âge espagnol, il fonde cette hypothèse risquée sur une dépendance formelle supposée des sculptures des églises hispaniques aux reliefs omeyyades de Syrie, affirmation qui va dans le sens de ce qu'avait avancé Sally Garen à la même époque, à savoir qu'il existait des correspondances entre la sculpture de Melque et celle du palais omeyyade de Khirbat al-Mafjar (GAREN, 1992 ; CABALLERO, 1992, 1994-1995, 1998, 2001 ; fig. 7). La voie de transmission aurait été le califat omeyyade de Cordoue, mais le raisonnement atteint ses 
limites dans l'absence de structures voûtées en pierre des édifices hispano-musulmans, alors qu'une tradition de voûtes est visible dans les églises wisigothiques ; leur datation fut donc retardée afin que l'ensemble du puzzle retrouve une cohérence (CABALLERO, 2000 ; UTRERO, 2006). Cette proposition découle d'une vision rigide de l'archéologie du bâti, élevée au rang de méthode pour la recherche, et qui fait généralement abstraction des données apportées par d'autres techniques de datation qui contredisent l'hypothèse de départ. L'archéologie du bâti se pose donc comme un modèle d'hypothèses à suivre et auxquelles il faut se tenir. Reste une question de base peu étudiée dans cette affaire de fixation des dates de construction à partir de la sculpture monumentale, à savoir celle de Khirbat al-Mafjar, qui est un édifice très classique, aussi classique que sa mosaïque ou que l'art wisigoth. Un bon motif de révision, d'ailleurs, pourrait être le fait que deux périodes - wisigothique et omeyyade - ont comme source d'inspiration commune l'Antiquité et ses arts : les palmettes de Melque sont héritières d'une tradition classique autant que celles de Syrie et sans forcément dépendre de celles-ci (PAZ, BELTRÁN, 2004). Selon un point de vue opposé, l'histoire de l'art et l'approche d'Isidro G. Bango Torviso (BANGO, 2001), entre autres, maintiennent les chronologies traditionnelles. Ainsi, face à une archéologie marquée par un développement méthodologique important à travers l'archéologie du bâti, l'histoire de l'art médiéval, étouffée entre des courants académiques conservateurs et le poststructuralisme, et une surinterprétation iconologique flagrante, semble forcée de se réinventer. Quoi qu'il en soit, aussi bien en archéologie qu'en histoire de l'art, il manque une révision fondamentale plus consciencieuse et moins partisane, ancrée dans des postures historiographiques contrastées (CARRERO, 2008).

Soulignant l'importance de la culture architecturale wisigothique, l'archéologie urbaine est peut-être celle qui offre les perspectives d'analyse les plus novatrices et l'avenir le plus prometteur (GURT, RIBERA, 2005 ; Recópolis..., 2006 ; GURT, SÁNCHEZ, 2008). Les travaux réalisés sur le terrain de la cathédrale de Valence rendent largement compte de la valeur cette approche : d'un édifice dont nous n'avions pratiquement plus que des fragments hors de tout contexte, on est parvenu à la reconstruction virtuelle d'une cathédrale en bonne et due forme (RIBERA, 2005). À Barcelone, il en va quasiment de même : quelques restes traditionnellement attribués à une basilique méconnue ont été réinterprétés comme les vestiges d'un lieu de passage liturgique dans un cadre urbain épiscopal, mais continuent à poser de sérieux problèmes d'interprétation (DUVAL, 1998 ; GODOY, 1998 ; GODOY, GURT, 1998 ; Bonnet, Beltrán DE Heredia, 2000 ; Beltrán De Heredia, 2001). Dans le cas de Recopolis, la nouvelle lecture de l'ensemble, rendue possible par des fouilles minutieuses, a renversé les interprétations d'usage sur cette ville fondée par Leovigild, sa topographie et sa reconstruction (Recópolis..., 2006 ; OLMO, 2008), bien que certains chercheurs continuent à douter que la ville identifiée comme Recopolis soit bien celle citée dans les textes.

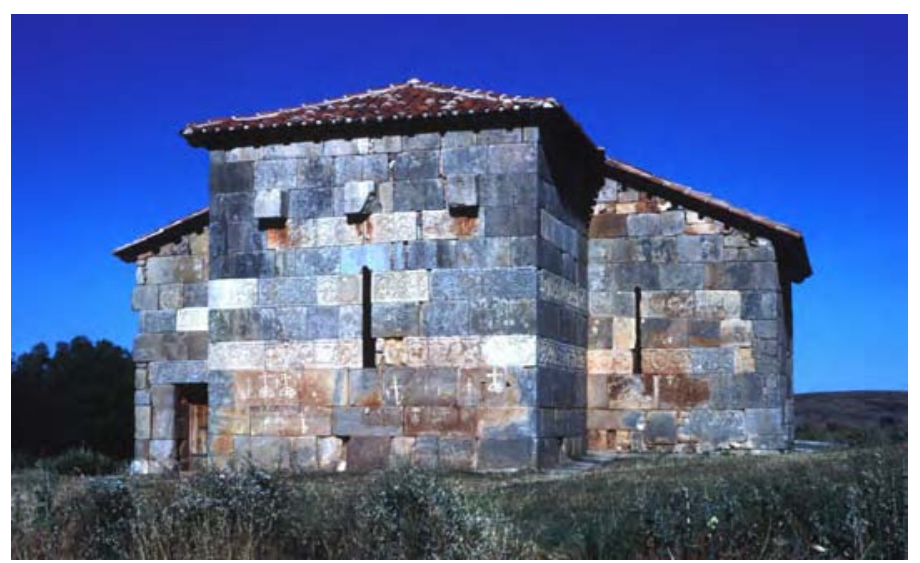

7. Chevet de l'église Quintanilla de las Viñas (Burgos). 
8a. Décor de chapiteau représentant le sacrifice d'Isaac par Abraham, église San Pedro de la Nave, Campillo (Zamora); b. extérieur de l'église.
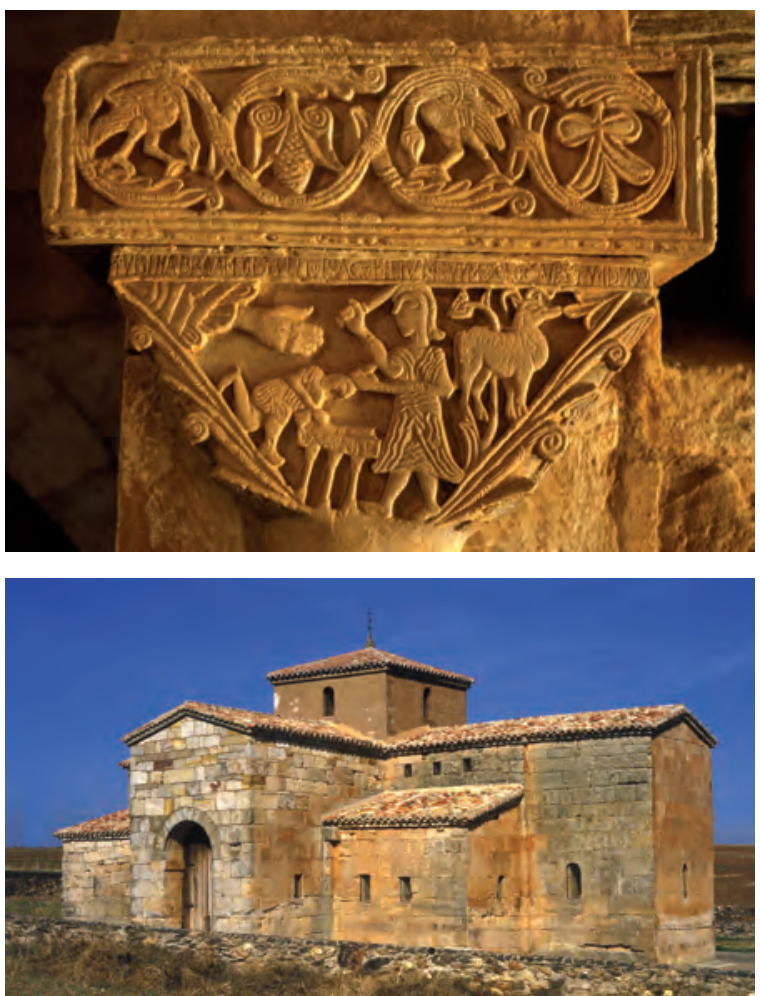

Nous ne pouvions terminer sans mentionner Tolède, la capitale inconnue et objet d'hypothèses fondées sur la documentation et sur une rare archéologie intramuros, ce à quoi les fouilles de la Vega Baja, actuellement en cours, se chargeront peut-être de remédier (RIPOLL, VELÁZQUEZ, 2000, 2008 ; IZQUIERDO, 2002 ; CARROBLES et al., 2008).

Le domaine de la sculpture, dans sa classification typologique, est toujours confronté à des difficultés de lecture stylistique et de catalogage, la méthode philologique ne semblant résoudre en rien les problèmes que le socle classique commun peut poser pour une sculpture, quelle qu'en soit la qualité. Son interprétation selon l'usage liturgique, et surtout l'iconographie, sont essentielles pour la relier au cadre et au système représentatif le plus adéquat (CRUZ, 1985 ; MORÍN DE PABlos, BARROSO, 2001 ; BARROSO, Morín DE PABLOS, 2007 ; fig. 8).

Enfin, un courant susceptible d'apporter de grandes et agréables surprises est celui de la tendance à l'étude de l'architecture à partir de la liturgie chrétienne et donc de la fonction des espaces architecturaux religieux. Cette ligne directrice historiographique, initiée par l'historiographie allemande et anglo-saxonne des siècles passés (WEDDIGEN, 2003), est ancienne mais relativement récente concernant les arts hispaniques. On compte quelques articles précurseurs de Miquel dels Sants Gros (GROS, 1988, 1992a, 1992b), mais c'est à Cristina Godoy que l'on doit la tentative d'utiliser cette approche comme objet de révision de l'architecture dite paléophrétienne : dans ses publications, la chercheuse a laissé entrevoir la richesse des possibilités qu'offre la nouvelle application de cette méthode (GODOY, 1989, 1992, 1995, 2004 ; continue à être nécessaire PUERTAS, 1975). Cependant, la méthode de Godoy - et non sa proposition de base - a été durement critiquée par Bango (BANGO, 1997) qui, en outre, a étendu la perspective chronologique de l'étude liturgique de l'époque hispano-wisigothique au XI ${ }^{\mathrm{e}}$ siècle, époque qui vit disparaître la liturgie hispanique en Occident péninsulaire, à la suite de l'officialisation de la liturgie gallo-romaine au concile de Burgos en 1080. Encore plus féroce fut la critique de Noël Duval (DUVAL, 2000), qui remit en question l'hypothèse de départ de Godoy en ce qui concerne le problème des contre-absides hispaniques, en relation à son usage dans le nord de l'Afrique (DUVAL, 2000 ; sa réponse dans GODOY, 2001). Dans la même lignée, une ébauche de réflexion a été publiée par Achim Arbeiter (ARBEITER, 2003). Malheureusement trop peu connue et en marge de cette polémique, on trouve la thèse d'Elena Quevedo-Chigas (QUEVEDO-CHIGAS, 2002), une appréciable mise à jour qui inclut un catalogage d'éléments architecturaux. Tout comme ce dernier livre, celui de Jordi Pinell, bien que fondamental, est également 
passé inaperçu (PINELL, 1998). Ce travail, trop peu connu, ouvre des perspectives qui conduisent Quevedo-Chigas à traiter non seulement l'architecture hispano-wisigothique mais aussi toute l'architecture qui, $\mathrm{du} \mathrm{V}^{\mathrm{e}}$ au $\mathrm{X}^{\mathrm{e}}$ siècle, fut érigée dans une même perspective : celle de la liturgie hispanique.

\section{L'accès au monde médiéval}

Cet état des lieux atteste la vitalité historiographique autour des problèmes que pose l'interprétation du monde hispano-wisigothique et de sa projection chronologique dans les trois premiers siècles après la disparition du royaume de Tolède. En rapport avec la liturgie, l'histoire de l'Occident péninsulaire, et en particulier la fondation du royaume des Asturies et son développement postérieur dans celui de León, a tendance à être considérée comme une unité issue de la volonté de restauration wisigothique, dont nous informent les chroniques asturiennes et qui - au moins d'un point de vue culturel - s'éteint au XI ${ }^{\mathrm{e}}$ siècle avec l'adoption de la liturgie gallo-romaine et l'arrivée des formes romanes. Ainsi donc, si dans les territoires de la Marca Hispanica il semble que la liturgie romaine se soit diffusée et imposée dès le IX ${ }^{e}$ siècle, l'histoire politique des territoires occidentaux de la péninsule aurait mené à sa conservation jusqu'aux environs de 1080, date officielle de sa substitution. C'est dans ce cadre chronologique des IX ${ }^{\mathrm{e}}$ et $\mathrm{X}^{\mathrm{e}}$ siècles que nous rencontrons les plus grandes complexités formelles (fig. 9). Pour quelles raisons ? La question se pose autour de deux paramètres fondamentaux : la continuité des formes ou leur renouvellement. Cette continuité s'est produite dans certains cas jusqu'au Bas Empire : c'est le cas des formes de l'art préroman asturien, qui, si elles furent, à une époque, considérées comme l'image provinciale du monde carolingien, sont interprétées aujourd'hui comme les héritières directes d'une Asturie romanisée, ainsi que l'archéologie tend à le démontrer ces dernières décennies (BANGO, 1988a, 1995, 2001 ; FERNÁNDEZ OCHOA et al., 2007). En revanche, pour l'art du Xe siècle - art de " repeuplement", art mozarabe, ou quel que soit le nom qu'on lui donne -, les axes de recherche oscillent entre deux positions. L'hypothèse traditionnelle, en allant plus loin qu'un simple problème terminologique, affirme qu'il est le fruit d'un enchevêtrement de formes où transparaîtraient clairement les influences hispano-musulmanes importées par ceux-là mêmes qui fuyaient le durcissement de leurs conditions de vie sous le gouvernement islamique au sud de la péninsule (GÓMEZ-MORENO, 1919 ; REGUERAS, 1990 ; BORRÁS, 1998 ; ARCE SÁINZ, 2001 ; MARTÍNEZ TEJERA, 2004). Selon l'autre hypothèse, l'art chrétien du $\mathrm{X}^{\mathrm{e}}$ siècle découlerait au contraire directement des formes hispanowisigothiques récupérées à la suite de la restauration des villes et des édifices religieux toujours debout après le repeuplement chrétien de la partie centre-nord de la

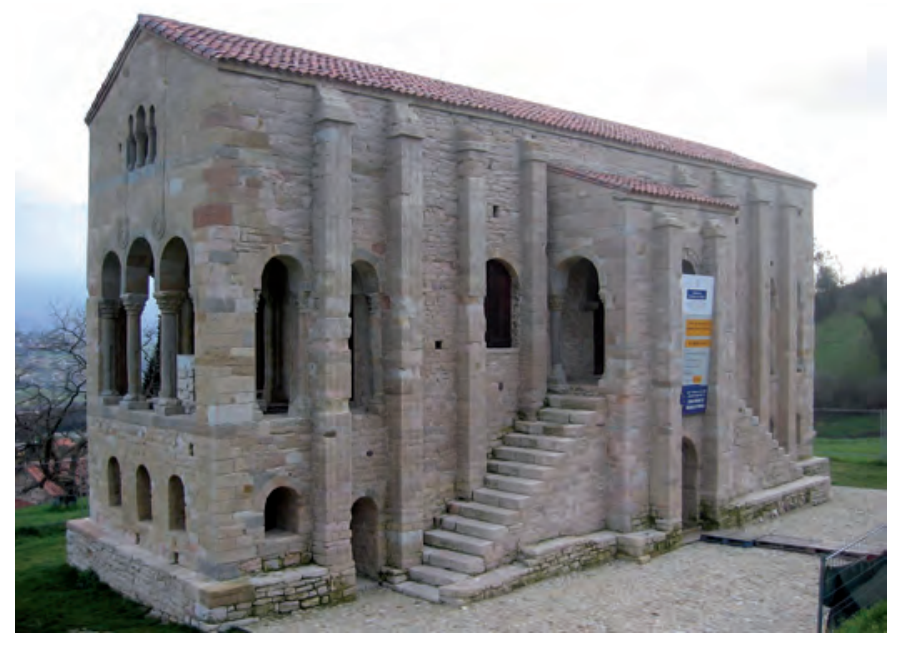

9. Santa María del Naranco (Oviedo), vers 841, édifice faisant partie d'un complexe architectural patronné par Ramiro I d'Oviedo. 
péninsule (CAMÓN, 1963 ; BANGO, 1979, 2007) 3. D'après cette thèse, seule l'arrivée de la culture de l'art roman a été capable de rompre avec la tradition en apportant un nouveau lexique constructif et ornemental.

Unité et différences, voilà le dilemme, si l'on reconnaît une ligne de continuité $\mathrm{du} \mathrm{V}^{\mathrm{e}} \mathrm{au} \mathrm{X}^{\mathrm{e}}$ siècle et, dans certains cas, jusqu'à un $\mathrm{XI}^{\mathrm{e}}$ siècle bien avancé. Il semble qu'au moins d'un point de vue cultuel et liturgique cette continuité s'impose. Mais dans une perspective formelle, la question se complique fortement si, comme on l'a vu précédemment, personne n'est d'accord sur la chronologie des édifices. Il est certes surprenant qu'une vingtaine d'édifices, circonscrits uniquement et exclusivement au monde hispanowisigoth, puisse faire couler tant d'encre et susciter des thèses si antagonistes. Heureusement, des perspectives s'ouvrent depuis que l'archéologie urbaine défriche les traces de ce brillant passé décrit dans les chroniques et les épigraphes, dévoilé par les bijoux et les ornamenta ecclesiae, mais qui, architecturalement, se voyait réduit à une poignée d'églises dans un environnement rural. On a redéfini Barcelone, Valence ou Terrassa, on continue à travailler sur Tolède et Carthagène, et d'autres lieux nous réservent sans doute des surprises... Stimulés par la vitalité de l'archéologie urbaine, serons-nous capables d'assumer de nouvelles positions et hypothèses, d'assimiler les changements potentiels, et, surtout, d'éliminer les barrières de la tradition et de la méthode qui asphyxient l'histoire matérielle du $V^{e}$ au $X^{\text {e }}$ siècle?

\section{Notes}

1. Enrique Flórez, España Sagrada. Theatro Geográphico-Histórico de la Iglesia de España. Origen, Divisiones y Términos de todas sus Provincias. Antigüedades, Traslaciones y Estudio antiguo de sus Sillas en todos los Dominios de España y Portugal. Con varias Dissertaciones críticas para ilustrar la Historia Eclesiástica de España, 29 vol., Madrid, 1747-1775. Cette œuvre colossale, restée inachevée, fut poursuivie par Manuel Risco (vol. 30 42) et par Antolín Merino et José de la Canal (vol. 43-46). Pedro Sainz de Baranda, Vicente de la Fuente, Carlos Ramón Fort, Eduardo Josué et Ángel Custodio Vega complétèrent l'España Sagrada en 56 volumes. La maison d'édition Editorial Augustiniana a commencé en 2000 la réédition de l'ensemble des volumes; trente en ont déjà été publiés et beaucoup sont consultables en ligne (principalement sur Google Books ou la Biblioteca Virtual Miguel de Cervantes).

2. Corpus Inscriptionum Latinarum II, Universidad de Alcalá de Henares, www2.uah.es/imagines_cilii.
3. Il s'est produit un phénomène similaire concernant les miniatures (YARZA, 1985) tandis que, en peinture, bien que cela ne concerne pas le champ de notre travail, quelques restes de polychromie retrouvés dans des églises du nord-ouest de la péninsule ont récemment été datés du $\mathrm{X}^{\mathrm{e}}$ siècle.

\section{Bibliographie}

- ÅBERG, 1922 : Nils Åberg, Die Franken und Westgoten in der Völkerwanderungszeit, Upsala, 1922.

- Alonso, 2005 : Raquel Alonso Álvarez, "Los enterramientos de los reyes visigodos", dans Fundamentos medievales de los particularismos hispánicos, (colloque, León, 2003), León, 2005, p. 361-376.

- Alonso, 2007 : Raquel Alonso Álvarez, "Los enterramientos de los reyes de León y Castilla hasta Sancho IV ", dans e-Spania, 3, juin 2007, publication électronique : http://e-spania.revues. org/index 109.html.

- Alonso, 2008 : Raquel Alonso Álvarez, "Hornija, Bamba, Pampliega: las elecciones funerarias de los reyes hispanovisigodos", dans Territorio, sociedad y poder: Revista de estudios medievales, 3, 2008, p. 13-27.

- ARBEITER, 2003 : Achim Arbeiter, "Los edificios de culto cristiano: escenarios de liturgia ", dans Pedro Mateos Cruz, Luis Caballero Zoreda éd., Repertorio de arquitectura cristiana en Extremadura: época tardoantigua y 
altomedieval, (Anejos del Archivo Español de Arqueología, 29), Mérida, 2003, p. 177-230.

- ARCE, 1982 : Javier Arce, El último siglo de la España romana (284-409 A.D.), Madrid, 1982.

- ARCE, 1988 : Javier Arce, España entre el mundo antiguo y el mundo medieval, Madrid, 1988.

- ARCE, 2005a : Javier Arce, "Antigüedad Tardía hispánica. Avances recientes ", dans Pyrenae: revista de prehistoria $i$ antiguitat de la Mediterrània occidental, 36/1, 2005, p. 7-32.

- ARCE, 2005b : Javier Arce, Bárbaros $y$ romanos en Hispania (400-507 A.D.), Madrid, 2005.

- ARCE SÁINZ, 2001 : Fernando Arce Sáinz, "Viejas y nuevas perspectivas sobre la cultura material mozárabe ", dans CABALLERO, MATEOS, 2001, p. 77-94.

- BALMASEDA, 1995 : Luis Javier Balmaseda Muncharaz, "El Tesoro perdido de Guarrazar ", dans Archivo Español de Arqueología, 68, 1995, p. 149-164.

- BALMASEDA, 1996 : Luis Javier Balmaseda Muncharaz, "Las versiones del hallazgo del Tesoro de Guarrazar ", dans Boletín del Museo Arqueológico Nacional, 14, 1996, p. 95-110.

- BANGo, 1979 : Isidro Gonzalo Bango Torviso, "Neovisigotismo artístico de los siglos IX y X: La restauración de ciudades y templos ", dans Revista de Ideas Estéticas, 148, 1979, p. 35-54.

- BANGo, 1985 : Isidro Gonzalo Bango Torviso, "L'Ordo Gothorum et sa survivance dans l'Espagne du Haut Moyen Âge ", dans Revue de l'Art, 70, 1985. p. 9-20.

- BANGo, 1988a : Isidro Gonzalo Bango Torviso, "Alfonso II y Santullano", dans Arte prerrománico y románico en Asturias, (colloque, Villaviciosa, 1984), Villaviciosa, 1988, p. 207-237.

- BANGo, 1988b : Isidro Gonzalo Bango Torviso, " El arte asturiano y el Imperio Carolingio ", dans Arte prerrománico y románico en Asturias, (colloque, Villaviciosa, 1984), Villaviciosa, 1988, p. 3188.

- BANGO, 1995 : Isidro Gonzalo Bango Torviso, " La cultura artística de la monarquía astur, la última manifestación de la Antigüedad ", dans Astures. Pueblos y cultura en la frontera del imperio romano, (cat. expo., Gijón, 1995), Gijón, 1995. p. 171-188.

- BANGO, 1997 : Isidro Gonzalo Bango Torviso, "La vieja liturgia hispana y la interpretación funcional del templo prerrománico ", dans José Ignacio de la Iglesia Duarte éd., VII Semana de Estudios Medievales, (colloque, Najéra, 1996), Logroño, 1997, p. 61-120.

- BANGO, 2001 : Isidro Gonzalo Bango Torviso, Arte prerrománico hispánico. El arte en la España cristiana de los siglos VI al XI, Madrid, 2001.

- BANGO, 2007 : Isidro Gonzalo Bango Torviso, «Un gravísimo error en la historiografía española: el empleo equivocado del término mozárabe ", dans Manuel Valdés Fernández éd., El legado de Al-Andalus. El arte andalusí en los reinos de León y Castilla durante la Edad Media, (colloque, León, 2006), León, 2007, p. 73-88

- BARATTE et al., 2008 : François Baratte et al., "Une question en débat: la transformation du monde romain et le rôle des barbares ", dans Perspective. La revue de l'INHA, 2008-1, p. 19-28.

- BARRoso et al., 1993: Rafael Barroso Cabrera et al., "El yacimiento de Tinto Juan de la Cruz (Pinto, Madrid). Algunas observaciones sobre el reparto de bienes y transformaciones de usos agrarios en época visigoda ", dans Rafael Azuar, Javier Martí Oltra éd., IV Congreso de Arqueología Medieval Española, Alicante, 1993, p. 297-298.

- BARRoso, MORIN DE PABlos, 2007 : Rafael Barroso Cabrera, Jorge Morín de Pablos, Regia Sedes Toletana, II, El Toledo visigodo a través de su escultura monumental, 2007.

- Beltrán De Heredia, 2001 : Julia Beltrán de Heredia Bercero éd., Los restos arqueológicos de la plaza del Rey de Barcelona: De Barcino a Barcinona (siglos I-VII), Barcelone, 2001.

- BIERBRAuer, 1994a : Volker Bierbrauer, "Archäologie und Geschichte der Goten vom 1.-7. Jahrhundert. Versuch einer Bilanz ", dans Frühmittelalterliche Studien, 28, 1994, p. 51-171.

- Bierbrauer, 1994b : Volker Bierbrauer, "Tracce archeologiche dei Visigoti fra il 376 e il 496-507 ", dans Ermanno A. Arslan, Volker Bierbrauer, Otto von Hessen éd., I Goti, Milan, 1994, p. 298-300.

- BIERBRAUER, 1999 : Volker Bierbrauer, " Les Wisigoths dans le royaume franc ", dans Françoise Vallet, Michel Kazanski, Patrick Périn éd., Des royaumes barbares au regnum francorum. L'Occident à l'époque de Childéric et de Clovis (vers 450vers 530), (colloque, Saint-Germain-enLaye, 1997), Paris, 1998, p. 167-200.
- BINFORD, 1971 : Lewis Roberts Binford, "Mortuary practices: Their Study and their potential ", dans American Archaeology, 25, 1971, p. 6-29.

- BINFORD, 1983 : Lewis Roberts Binford, In Pursuit of the Past. Decoding the Archaeological Record, Londres, 1983.

- BonNet, Beltrán DE HeREdia, 2000 : Charles Bonnet, Julia Beltrán de Heredia Bercero, "Nuevas intervenciones arqueológicas en el Museo de Historia de la Ciudad: Una iglesia de época visigótica en el grupo episcopal de Barcelona ", dans $V$ Reunión de Arqueología Cristiana Hispánica, (colloque, Carthagène, 1998), Carthagène, 2000, p. 135-144.

- BORRÁs, 1998 : Gonzalo Máximo Borrás Gualis, "Revisión crítica de los estudios de don José Camón Aznar (1898-1979) sobre el arte musulmán y mudéjar, arte mozárabe y arte del Renacimiento ", dans Boletín del Museo e Instituto Camón Aznar, 72, 1998, p. 89-98.

- BOWERSOCK, BROWN, GRABAR, 1999 : Glen Warren Bowersock, Peter Brown, Oleg Grabar éd., Late Antiquity: A Guide to the Postclassical World, Cambridge (MA), 1999.

- BOWERSOCK, BROWN, GRABAR, 2001 : Glen Warren Bowersock, Peter Brown, Oleg Grabar éd., Interpreting Late Antiquity: Essays on the Postclassical World, Cambridge (MA), 2001.

- Brown, 1971 : Peter Brown, The World of Late Antiquity: From Marcus Aurelius to Muhammad, Londres, 1971.

- BRown, 1978 : Peter Brown, The Making of Late Antiquity, Cambridge, (MA), 1978.

- Brown, 1981 : Peter Brown, The Cult of the Saints: Its Rise and Function in Latin Christianity, Chicago, 1981.

- Brown, 1982 : Peter Brown, Society and the Holy in Late Antiquity, Londres, 1982.

- BROWN, 1996 : Peter Brown, The Rise of Western Christendom: Triumph and Diversity, A.D. 200-1000, Cambridge (MA)/ Oxford, 1996.

- Brown et al., 1997 : Peter Brown et al., "The Worlds of Late Antiquity Revisited ", dans Symbolae Osloenses, 72 , 1997, p. 5-90.

- CABALLERO, 1992 : Luis Caballero Zoreda, « Visigodo o asturiano? Nuevos hallazgos en Mérida y otros datos para un nuevo marco de referencia de la arquitectura y la escultura altomedieval en el Norte y Oeste de la Península Ibérica ", dans XXXIX Corso di Cultura sull'Arte Ravennate 
e Bizantina, Ravenne, 1992, p. 139-189. - CABAllero, 1994-1995 : Luis Caballero Zoreda, "Un canal de transmisión de lo clásico en la alta Edad Media española. Arquitectura y escultura de influjo omeya en la Península Ibérica entre mediados del s. VIII e inicios del X ", dans Al-Qantara, 15, 1994 p. 321-348 et 16, 1995, p. 107-124 - CABALLERO, 1998 : Luis Caballero Zoreda, "Arquitectura visigótica y musulmana: continuidad, concurrencia o innovación?", dans Ruptura o continuidad pervivencias preislámicas en Al-Ándalus, Cuadernos Emeritenses, 15, 1998, p. 143-176. - CABAllero, 2000 : Luis Caballero Zoreda, "Posibilidades de la arqueología de la arquitectura. A propósito de la arquitectura abovedada altomedieval ", dans Amparo Graciani Garcia éd., Actas del tercer Congreso Nacional de Historia de la construcción, (colloque, Séville, 2000), Séville, 2000, p. 125-134.

- CABAllero, 2001 : Luis Caballero Zoreda, « La arquitectura denominada visigoda, ¿es realmente tardorromana o prerrománica?", dans CABALLERO, MATEOS, 2001, p. 207-248.

- CABALleRo, 2007 : Luis Caballero Zoreda, "Producción decorativa y estratigrafía ", dans Luis Caballero Zoreda, Pedro Mateos Cruz éd., Escultura decorativa tardorromana y altomedieval en la Península Ibérica, (Anejos de Archivo Español de Arqueología, 41), Madrid, 2007, p. 233-274. - Caballero, Mateos, 2001 : Luis Caballero Zoreda, Pedro Mateos Cruz éd., Visigodos y Omeyas. Un debate entre la Tardo Antigüedad y la Alta Edad Media, (colloque, Mérida, 1999), (Anejos de Archivo Español de Arqueología, 23), Madrid, 2001.

- CAILlet, 1985 : Jean-Pierre Caillet, L'Antiquité classique. Le haut Moyen Âge et Byzance au Musée de Cluny, Paris, 1985.

- CAILlet, 2001 : Jean-Pierre Caillet, "Alois Riegl et le fait social dans l'art de l'Antiquité tardive", dans Antiquité Tardive, 9, 2001, p. 47-51.

- CAMERON, 1993 : Averil Cameron, The Mediterranean World in Late Antiquity, 395-600, Londres, 1993.

- CAMÓn, 1963 : José Camón Aznar "Arquitectura española del siglo X. Mozárabe y de la Repoblación ", dans Goya, 52, 1963, p. 206-219.

- CAMPs, 1940 : Emilio Camps Cazorla, "El arte hispano-visigodo", dans Ramón Menéndez Pidal éd., Historia de España, III, España visigoda, Madrid, 1940, p. 435-608.
- Cardelle de Hartmann, 2001 : Carmen Cardelle de Hartmann, Victoris Tvnnvnensis Chronicon cum reliquiis ex Consularibus Caesaraugustanis et Iohannis Biclarensis Chronicon, (Collection Corpus Christianorum, Series Latina, 173 A), Turnhout, 2001.

- CARrero, 2008 : Eduardo Carrero Santamaría, "Teoría y método en la Historia de la arquitectura medieval. Algunas reflexiones ", dans Guillem Rosselló Bordoy, Francesca Tugores Truyol éd., Seminari d'Estudis històrics 2007: Arqueologia de l'Arquitectura (colloque, Palma de Majorque, 2007), Palma de Majorque, 2008, p. 5-27.

- CARRobles et al., 2007 : José Carrobles et al., Regia Sedes Toletana, 2 vol., Tolède, 2008.

- Caveda y Nava, 1848 : José Caveda y Nava, Ensayo histórico sobre los diversos géneros de arquitectura empleados en España desde la dominación romana hasta nuestros días, Madrid, 1848.

- CHAVARríA, 2007 : Alexandra Chavarría, El final de las villae en Hispania (siglos IV-VII d.C.), (Bibliothèque de l'Antiquité Tardive, 7), Turnhout, 2007.

- CID, 1980 : Carlos Cid Priego, " ¿Existió miniatura prerrománica asturiana? ", dans Liño: revista anual da historia del arte, 1, 1980, p. 107-142.

- Collins, 2004 : Roger Collins, Visigothic Spain (409-711), Malden, 2004. - CRUZ, 1985 : Maria Cruz Villalón, Mérida Visigoda: La Escultura Arquitectónica y Litúrgica, Badajoz, 1985.

- Duval, 1992 : Noël Duval, "PaulAlbert Février (1931-1991) et Charles Pietri (1932-1991) : Deux espécialistes d'archéologie et d'histoire de l'Antiquité tardive ", dans Archivo Español de Arqueología, 65, 1992, p. 291-297. - Duval, 1998 : Noël Duval, "La cathédrale de Barcelone revisitée ", dans Bulletin Monumental, 156, 1998, p. 403-410. - DuvAL, 2000 : Noël Duval, « Les relations entre l'Afrique et l'Espagne dans le domaine liturgique: existe-t-il une explication commune pour les 'contre-absides' et 'contre-chœurs' ? À propos de Cristina Godoy Fernández, Arqueología y Liturgia, Barcelona, 1995 ", dans Rivista di Archeologia Cristiana, 76, 2000, p. 429-476.

- FERnÁNDEZ GUERRA, 1866 : Aureliano Fernández Guerra, "Inscripciones Cristianas y antiguos monumentos del arte Cristiano Español ", dans El Arte en España, 4, 1866, p. 73-87.
- Fernández OchoA et al., 2007 : Carmen Fernández Ochoa, Fernando Gil Sendino, Javier Del Hoyo, "Una inscripción y un dibujo sobre el ladrillo hallados en la villa romana de Veranes (Gijón, Asturias) ", dans Archivo Español de Arqueología, 80, 2007, p. 183 190.

- FERNÁNDEZ-ORDÓÑEZ, 2000 : Inès Fernández-Ordóñez éd., Alfonso X el Sabio y las crónicas de España, Valladolid, 2000.

- Fernández SomozA, 2004 : Gloria Fernández Somoza, « La Biblia de León del año 920 en el contexto de la miniatura hispánica ", dans José Joaquin Yarza, Maria Victoria Herráez, Gerardo Boto éd., La catedral de León en la Edad Media, (colloque, León, 2003), León, 2004, p. 499-507.

- FONTAINE, 1973 : Jean Fontaine, L'art pré-roman hispanique, I, Les arts paléochrétien, wisigothique, asturien, Abbaye de la Pierre-qui-Vire, 1973.

- FonTAINE, 1977 : Jean Fontaine, L'art pré-roman hispanique, II, L'art mozarabe, Abbaye de la Pierre-qui-Vire, 1977.

- García, Moro, Tuset, 2009 : Gemma Garcia Llinares, Antonio Moro García, Francesc Tuset Bertran, La seu episcopal d'Ègara. Arqueologia d'un conjunt cristià del segle IV al IX, (Institut Català d'Arqueologia Clàssica), Barcelone, 2009.

- GAREN, 1992 : Sally Garen, "Santa María de Melque and Church Construction under Muslim Rule ", dans Journal of Society of Architectural Historians, 51/ 2, 1992, p. 288-305.

- GIBBON, 1776-1789 : Edward Gibbon, History of the Decline and Fall of the Roman Empire, I, 1776 ; II, III, 1781 ; IV, V, VI, 1788-1789.

- GIARDINA, 1999 : Andrea Giardina, "Esplosione di tardoantico ", dans Stu di Storici, 40/1, 1999, p. 157-180.

- Gimeno, 2004 : Helena Gimeno Pascual, " La epigrafía en San Pedro de la Nave ", dans Luis Caballero Zoreda éd., La iglesia de San Pedro de la Nave (Zamora), Zamora, 2004, p. 239-273.

- GODOY, 1989 : Cristina Godoy Fernández, "Arquitectura cristiana y liturgia: reflexiones en torno a la interpretación funcional de los espacios ", dans Espacio, Tiempo y Forma, Serie I: Prehistoria y arqueología, II, 1989, p. $355-$ 388 .

- GODOY, 1992 : Cristina Godoy Fernández, "Arqueología cristiana y liturgia ", dans Gisela Ripoll López éd., Arqueología hoy, Madrid, 1992, p. 153-162. 
- Godoy, 1995 : Cristina Godoy Fernández, Arquitectura y liturgia. Iglesias hispánicas (siglos IV-VIII), Barcelone, 1995.

- Godoy, 1998 : Cristina Godoy Fernández, «El complejo episcopal de Barcino. Cuestiones sobre la función e identificación de los edificios ", dans Madrider Mitteilungen, 39, 1998, p. 311 335.

- Godoy, 2001 : Cristina Godoy Fernández, "Sobre arqueología y liturgia en las iglesias hispanas. Breve respuesta a la recensión de N. Duval ", dans Rivista di Archeologia Cristiana, 77, 2001 , p. 469-480.

- Godoy, 2004 : Cristina Godoy Fernández, "A los pies del templo. Los espacios litúrgicos en contraposición al altar: una revisión ", dans José Maria Blázquez, Antonio González Blanco éd., Antigüedad y Cristianismo. Monografías históricas sobre la Antigüedad tardía. Ejemplar dedicado a: Sacralidad y arqueología. Homenaje al profesor Thilo Ulbert al cumplir 65 años, 21, 2004, p. 473-492.

- GODOY, GURT, 1998 : Cristina Godoy Fernández, Josep Maria Gurt Esparraguera, "Un itinerario de peregrinaje para el culto martirial y veneración del agua bautismal en el complejo episcopal de Barcino ", dans Madrider Mitteilungen, 39, 1998, p. 323-335.

- GOETZ, JARNUT, POHL, 2003 : HansWerner Goetz, Jörg Jarnut, Walter Pohl éd., Regna and Gentes. The Relationship between Late Antique and Early Medieval Peoples and Kingdoms, (The Transformation of the Roman World, 13), Leyde/ Boston, 2003.

- GÓMEZ-MORENO, (1889) 1988 : Manuel Gómez-Moreno, Monumentos romanos y visigóticos de Granada, Grenade, (1889) 1988.

- GÓMEZ-MORENO, 1906 : Manuel Gómez-Moreno, "San Pedro de La Nave. Iglesia visigoda ", dans Boletín de la Sociedad Castellana de Excursiones, 4, 1906, p. 3-11.

- Gómez-Moreno, 1919 : Manuel Gómez-Moreno, Iglesias mozárabes. Arte español de los siglos IX a XI, Madrid, 1919 (rééd. facs., Grenade, 1998).

- GÖTZE, 1907 : Alfred Götze, Götische Schnallen, Berlin, 1907.

- GROS, 1988 : Miquel dels Sants Gros i Pujol, "Fiesta y liturgia en el Liber Ordinum hispánico ", dans Alfonso de Esteban Alonso, Jean-Pierre Etienvre éd., Fiestas y liturgia. Actas del coloquio celebrado en la Casa de Velázquez, Madrid, 1988, p. 11-20.

- GROS, 1992a : Miquel dels Sants Gros i Pujol, "Las dificultats del coneixement des de la litúrgia ", dans Actuacions del patrimoni edificat: la restauració de l'arquitectura dels segles IX $i X$ (investigació històrica $i$ disseny arquitectònic), (colloque, Barcelone, 1991), Barcelone, 1992, p. 31-34.

- GROS, 1992b : Miquel dels Sants Gros i Pujol, " La funcionalitat litúrgica de les esglésies d'Egara ", dans Simposi internacional sobre les Esglésies de Sant Pere de Terrassa, (colloque, Terrassa, 1991), Terrassa, 1992, p. 77-83.

- GURT, GUARDiA, RIPOLL, 2005 : Josep Maria Gurt Esparraguera, Milagros Guardia, Gisela Ripoll, "Pere de Palol i Salellas ", dans Pyrenae, 36/2, 2005 , p. 5-7.

- GURT, RiBERA, 2005 : Josep Maria Gurt Esparraguera, Albert Ribera i Lacomba, VI Reunió d'Arqueologia Cristiana Hispánica. Les ciutats tardoantigues d'Hispania: Cristianització $i$ topografía, (colloque, Valence, 2003), Barcelone, 2005.

- GURT, SÁNCHEZ, 2008 : Josep Maria Gurt Esparraguera, Isabel Sánchez Ramos, "Las ciudades hispanas durante la antigüedad tardía: una lectura arqueológica ", dans OLmo, 2008, p. 183-202.

- HAmerow, 2002 : Helena Hamerow, Early Medieval Settlements: The Archaeology of Rural Communities in North-West Europe 400-900, Oxford, 2002.

- HÄRKE, 1992 : Heinrich Härke, "Changing Symbols in Changing Society: The Anglo-Saxon Weapon Burial Rite in the Seventh Century ", dans Martin Carver éd., The Age of Sutton Hoo, Woodbridge, 1992, p. 149-165.

- HeAther, 1996 : Peter Heather, The Goths, Oxford/Cambridge (MA), 1996.

- Hilaire, 1999 : Yves-Marie Hilaire éd., De Renan à Marrou. L'histoire du christianisme et les progrès de la méthode historique (1863-1968), Villeneuve d'Ascq, 1999.

- HOdDER, 1986 : Ian Hodder, Reading the Past. Current approaches to interpretation in Archaeology, Cambridge, 1986. - HOWARD-JOHNSTON, HAYWARD, 1999 : James Howard-Johnston, Paul Antony Hayward éd., The Cult of Saints in Late Antiquity and the Early Middle Ages: Essays on the Contribution of Peter Brown, Oxford, 1999.
- ÍÑIGUEZ, 1953 : Francisco Íñiguez Almech, "Algunos problemas de las viejas iglesias españolas ", dans Cuadernos de trabajos de la Escuela Española de Historia y Arqueología de Roma, 7, 1955, p. 9-180. - IZQUIERDO, 2001 : Ricardo Izquierdo Benito, "Alfonso X el Sabio, ¿primer arqueólogo medievalista? ", dans Historia, Instituciones, Documentos, 28, 2001, p. 231-240.

- IzQUIERDO, 2002 : Ricardo Izquierdo Benito, "Toledo en época visigoda ", dans Toledo y Bizancio, Cuenca, 2002, p. 43-74.

- JAMES, 2008 : Edward James, "The Rise and Function of the Concept Late Antiquity ", dans Journal of Late Antiquity, 1/1, 2008, p. 20-30.

- Jiménez GARNiCA, 1985 : Ana Maria Jiménez Garnica, " El origen de la Legislación Civil Visigoda sobre la prohibición de matrimonios entre romanos y godos: Un problema de fundamento religioso ", dans Anuario de Historia del Derecho Español, 1985, p. 735-747.

- Jiménez GARnicA, 1999 : Ana Maria Jiménez Garnica, "Settlement of the Visigoths in the Fifth Century ", dans Peter Heather éd., The Visigoths from the Migration Period to the Seventh Century. An Ethnographic Perspective, Woodbridge, 1999, p. 93-128.

- JONES, 1964 : Arnold Hugh Martin Jones, The Later Roman Empire, AD 284602: A Social, Economic and Administrative Survey, 2 vol., Oxford, 1964.

- KAPUŚCIŃSKI, 2005 : Ryszard Kapuściński, "Encountering the Other: The Challenge for the $21^{\text {st }}$ Century ", dans New Perspectives Quarterly, 22/4, 2005, p. 6-13.

- KAZANSKI, 1985 : Michel Kazanski, "Le peigne en os ", dans Mary Larrieu et al., La nécropole mérovingienne de la Turraque à Beaucaire-sur-Baïse (Gers), Toulouse, 1985, p. 257-269.

- KAZANSKI, LAPART, 1995 : Michel Kazanski, Jacques Lapart éd., "Quelques documents $d u V^{e}$ siècle ap. J.-C. attribuables aux Wisigoths découverts en Aquitaine ", dans Aquitania, 13, 1995 , p. 193-202.

- KocH, 2006 : Manuel Koch, "Gotthi intra Hispanias sedes acceperunt. Consideraciones sobre la supuesta inmigración visigoda en la Península Ibérica ", dans Pyrenae, 37/2, 2006, p. 83-104.

- KULiKowsKi, 2004 : Michael Kulikowski, Late Roman Spain and Its Cities, Baltimore/Londres, 2004. 
- LA RocCA, 1989 : Christina La Rocca, "Le sepolture altomedievali del territorio di Verona ", dans Materiali di età longobarda nel veronese, Denise Modonest, Christina La Rocca éd., (cat. expo., Vérone, Museo di Castelvecchio, 1989), Vérone, 1989, p. 149-180.

- LAMPÉREZ Y ROMEA, (1908-1909) 1999 : Vicente Lampérez y Romea, Historia de la arquitectura cristiana española en la Edad Media, según el estudio de los elementos y los monumentos, (Madrid, 1908-1909) Valladolid, 1999.

- LiebeschuetZ, 1998 : John H. W. G. Liebeschuetz, "Citizen status and law in the roman Empire and the visigothic Kingdom ", dans POHL, REIMITZ, 1998, p. 131-152.

- LiebeschuetZ, 2001 : John H. W. G. Liebeschuetz, The Decline and Fall of the Roman City, Oxford/New York, 2001. - LÓPEZ SERRANO, 1963 : Matilde López Serrano, "Arte visigodo: Arquitectura y escultura. Artes decorativas de la época visigoda. Adiciones ", dans Ramon Menéndez Pidal éd., Historia de España, III, España visigoda, $2^{\mathrm{e}}$ éd., Madrid, 1963, p. 725-830.

- MAier AlLende, 2006 : Jorge Maier Allende, "Aureliano Fernández-Guerra, Giovanni Battista de Rossi y la arqueología paleocristiana en la segunda mitad del siglo XIX ", dans José Beltrán Fortes, Beatrice Cacciotti, Beatrice Palma Venetucci éd., Arqueología, coleccionismo y antigüedad: España e Italia en el siglo XIX, (colloque, Séville, 2004), Séville, 2006, p. 299-349.

- MARrou, 1938 : Henri-Irénée Marrou, Saint Augustin et la fin de la culture antique, 2 vol., Paris, 1938.

- MARRoU, 1968 : Henri-Irénée Marrou, "La civilisation de l'Antiquité tardive" dans Atti del Convegno internazionale Tardo Antico e Alto Medioevo, La forma critica nel passaggio dell'antichità al medioevo, (colloque, Rome, 1967), (Problemi attuali di scienza e di cultura, 105), Rome, 1968.

- MARrou, 1977 : Henri-Irénée Marrou, Décadence romaine ou antiquité tardive? $I I I^{e}-I V^{e}$ siècle, Paris, 1977.

- MARrou, 1978 : Henri-Irénée Marrou, Christiana Tempora. Mélanges d'histoire, d'archéologie, d'épigraphie et de patristique, Rome, 1978.

- MARTIN, 1976 : René Martin, "Qu'est-ce que l'Antiquité 'tardive' ? Réflexions sur un problème de périodisation ", dans Raymond Chevalier éd. Aiôn. Le temps chez les romains, (Collec- tion Caesarodunum, 10bis), Paris, 1976 , p. 261-304.

- MARTIN, 1991 : Max Martin, « Zur frühmittelalterlichen Gürteltracht der Frau in der Burgundia, Francia und Aquitania ", dans L'art des invasions en Hongrie et en Wallonie, (colloque, Mariemont, 1979), Mariemont, 1991, p. 31-85.

- MARtínez Tejera, 2004：Artemio Manuel Martínez Tejera, « La arquitectura cristiana hispánica de los siglos IX y x en el Regnum astur-leonés ", dans Argutorio, 14, 2004, p. 9-12.

- Molina Gómez, 2002 : José Antonio Molina Gómez, "Henri Irénée Marrou (1904-1977) ", dans Antigüedad y Cristianismo, 19, 2002, p. 379-397.

- Molist, Gurt, PAlol, 2002 : Miquel Molist Montanà, Josep Maria Gurt i Esparraguera, Pere de Palol i Salellas, Premi d'Arqueologia Catalana. Pàtera d'honor 2002 a Pere de Palol i Salellas, Barcelone, 2002.

- Morín de PABlos, BARroso, 2001 : Jorge Morín de Pablos, Rafael Barroso Cabrera, "Fórmulas y temas iconográficos en la plástica hispanovisigoda (siglos VI-VIII): El problema de la influencia oriental en la cultura material de la España tardoantigua y altomedieval ", dans CABALlero, MATEOS, 2001 , p. 279-306

- Morín De PABlos, BARRoso, 2005 : Jorge Morín de Pablos, Rafael Barroso Cabrera, "El mundo funerario de época visigoda en la Comunidad de Madrid. El poblamiento y el problema del asentamiento de los visigodos en la Península Ibérica a través del estudio de las necrópolis madrileñas", dans Actas de las Primeras Jornadas de Patrimo nio Arqueológico en la Comunidad de Madrid, (colloque, Madrid, 2004), Madrid, 2005, p. 183-213.

- Olmo, 1991 : Lauro Olmo Enciso, "Ideología y Arqueología: los estudios sobre el periodo visigodo en la primera mitad del siglo XX ", dans Ricardo Olmos Romera, Javier Arce Martínez éd. Historiografía de la Arqueología y de la Historia Antigua de España (siglos XVIII$X X)$, (colloque, Madrid, 1988), Madrid, 1991, p. 157-160.

- Olmo, 2008 : Lauro Olmo Enciso éd., Recópolis y la ciudad en la época visigoda numéro thématique de Zona arqueológica, 9, 2008.

-PALANQUe, 1978 : Jean-Rémy Palanque, "Notice sur la vie et les tra- vaux de Henri-Irénée Marrou ", dans Comptes rendus de l'Académie des Inscriptions et Belles-Lettres, Paris, 1978, p. 401 418.

- PAlol, 1956 : Pedro de Palol, "Esencia del arte hispánico de época visigoda: romanismo y germanismo ", dans I goti in occidente, problemi, Settimane di Studio del Centro Italiano di Studi sull'Alto Medioevo, Spolète, 1956, p. 65-126.

- PAlol, 1967 : Pedro de Palol, Arqueología cristiana de la España romana, siglos IV-VI, Madrid, 1967.

- PAlol, (1940) 1991 : Pedro de Palol, "Arte y Arqueología ", dans Rámon Menéndez Pidal éd., Historia de España III/2, España visigoda. La Monarquía. La Cultura. Las Artes, Madrid, (1940) 1991, p. 269-428.

- PAZ, Beltrán, 2004 : Juan Angel Paz Peralta, Miguel Beltrán Lloris, "La transmisión decorativa a través de los emblemas militares, desde la Antigüedad clásica a la Edad Media: la escultura decorativa en Aragón desde el siglo VII al año 1030 ", dans Boletín del Museo de Zaragoza, 18, 2004, p. 79-238.

- Perea, 2001 : Alicia Perea, El tesoro visigodo de Guarrazar, Madrid, 2001.

- Pinell Pons, 1998 : Jordi Pinell Pons, Liturgia hispánica, Barcelone, 1998.

- PIRENNe, 1937 : Henri Pirenne, Mahomet et Charlemagne, Paris/Bruxelles, 1937. - PoHL, 1997 : Walter Pohl éd. Kingdoms of the Empire: The Integration of Barbarians in Late Antiquity, Leyde/ New York/ Köln/Brill, 1997.

- PoHL, 2000a : Walter Pohl, Le origini etniche dell'Europa. Barbari e Romani tra antichità e medioevo, Rome, 2000.

- POHL, 2000b : Walter Pohl, Die Germa nen, Munich, 2000.

- Pohl, Reimitz, 1998: Walter Pohl, Helmut Reimitz éd., Strategies of Distinc tion: The Construction of Ethnic Communities, 300-800, Leyde/Boston, 1998.

- Puertas, 1975 : Rafael Puertas Tricas, Iglesias hispánicas (siglos IV al VIII). Testimonios literarios, Madrid, 1975.

- PUIG I CADAFALCH, 1936a : Josep Puig i Cadafalch, La basílica de Tarragona: períodes paleocristià $i$ visigòtic, Barcelone, 1936.

- PUig I CADAFAlCh, 1936b : Josep Puig i Cadafalch, La sèu visigòtica d'Egara. Barcelone, 1936.

- PUig I CADAfAlch, 1948 : Josep Puig i Cadafalch, Noves descobertes a la catedral d'Egara. La catedral primitiva, la catedral visigótica, les pintures de la segona catedral, Barcelone, 1948. 
- PUig I CADAfAlch, 1961 : Josep Puig i Cadafalch, L'Art wisigothique et ses survivances. Recherches sur les origines et le développement de l'Art en France et en Espagne du IV au XII siècle, Paris, 1961.

- Quevedos-Chigas, 2002 : Elena Quevedo-Chigas, Early medieval Iberian architecture and the Hispanic liturgy: a stu$d y$ of the development of church planning from the fifth to the tenth centuries, Michigan, 2002.

- Recópolis..., 2006 : Recópolis. Un paseo por la ciudad visigoda, Lauro Olmo Enciso éd., (cat. expo., Madrid, Museo Arqueológico Regional de la Comunidad de Madrid, 2006), Madrid, 2006.

- REgueras, 1990 : Fernando Regueras, La arquitectura mozárabe en León y Castilla, Valladolid, 1990.

- REINHART, 1945 : Wilhelm M. Reinhart, "Sobre el asentamiento de los visigodos en la Península ", dans Archivo Español de Arqueología, 18, 1945, p. 124139.

- RIBERA, 2005 : Albert Ribera i Lacomba, "Origen i desenvolupament del nucli episcopal de València ", dans GURT, RIBERA, 2005, p. 207-244.

- RICHARDS, 1995 : Julian D. Richards, "An Archaeology of Anglo-Saxon England ", dans Giorgio Ausenda éd., After Empire. Towards an Ethnology of Europe's Barbarians, (Studies in Historical Archaeoethnology, 1), San Marino, 1995, p. 51-74.

- RICHÉ, 2003 : Pierre Riché, Henri Irénée Marrou. Historien engagé, Paris, 2003. - RIEGL, 1901-1923 : Alois Riegl, Spätrömische Kunstindustrie nach den Funden in Österreich-Ungarn, Vienne, I, 1901 ; II, 1923.

- Ríos, 1861 : José Amador de los Ríos, El arte latino bizantino en España y las coronas visigodas de Guarrazar: ensayo histórico crítico, Madrid, 1861.

- RIPOLL, 1983 : Gisela Ripoll, «Entrevista con el Prof. Pedro de Palol", dans Revista de Arqueología, 31, 1983, p. $42-47$.

- RIPOLL, 1989 : Gisela Ripoll, « Historiografía y numismática visigodas entre los siglos XVI y XVIII: Ambrosio de Morales y Enrique Flórez", dans Archéologie aujourd'hui. Les Mérovingiens, archéologie et historiographie, (Collection Archéologie aujourd'hui, 2), Paris, 1989, p. 58-64.

- RIPOLL, 1991 : Gisela Ripoll, "Materiales funerarios de la Hispania visigoda: problemas de cronología y tipología ", dans Patrick Périn éd., Gallo-romains, Wisigoths et Francs en Aquitaine, Septimanie et Espagne, (colloque, Toulouse, 1985), Rouen, 1991, p. 111-132.

-RIPOLL, 1993-1994 : Gisela Ripoll, "La necrópolis visigoda de El Carpio de Tajo. Una nueva lectura a partir de la topocronología y los adornos personales ", dans Butlletí de la Reial Acadèmia Catalana de Belles Arts de Sant Jordi, 7/8, Barcelone, 1993-1994, p. 187-250.

- RIPOLL, 1998 : Gisela Ripoll, "The arrival of the Visigoths in Hispania: population problems and the process of acculturation ", dans POHL, REIMITZ, 1998, p. 153-187.

- RIPOLL, 1999 : Gisela Ripoll, "Symbolic Life and Signs of Identity in Visigothic Times", dans Peter Heather éd., The Visigoths from the Migration Period to the Seventh Century: An Ethnographic Perspective, (Studies in Historical Archeoethnology, 4), Woodbridge, 1999. p. 403-431.

- RIPOLL, 2000 : Gisela Ripoll, « Visigothic Jewelry of the Sixth and Seventh Centuries ", dans Katherine R. Brown, Dafydd Kidd, Charles T. Little éd., From Attila to Charlemagne. Arts of the Early Medieval Period in the Metropolitan Museum of Art, New York/New Haven, 2000, p. 188-203.

- RIPOLL, 2001a : Gisela Ripoll, "Problemas cronológicos de los adornos personales hispánicos (finales del siglo V-inicios del VIII)", dans Javier Arce Martínez, Paolo Delogu éd., Visigoti e Longobardi, Florence, 2001, p. 5777.

- RIPOLL, 2001b : Gisela Ripoll, "Romani e Visigoti in Hispania: problemi di interpretazione del material archeologico ", dans Paolo Delogu éd., Le invasione barbariche nel meridione dell' Impero: Visigoti, Vandali, Ostrogoti, Cosenza, 2001, p. 99-117.

-RIPOLL, 2004 : Gisela Ripoll, " Il Tesoro di Guarrazar. La tradizione dell'oreficeria nella tarda antichità ", dans Sauro Gelichi, Cristina La Rocca éd., Tesori. Forme di accumulazione della ricchezza nell'alto medioevo (secoli V-XI), Rome, 2004, p. 207-240.

- RIPOLL, 2006 : Gisela Ripoll, "Las necrópolis visigodas. Reflexiones en torno al problema de la identificación del asentamiento visigodo en Occidente según los materiales arqueológicos", dans Hispania Gothorum. San Ildefonso y el reino visigodo de Toledo, (cat. expo., Tolède, Museo de Santa Cruz de Toledo, 2006), Tolède, 2006, p. 59-74.

- RIPOLL, sous presse : Gisela Ripoll, "The Archaeological Characterisation of the Visigothic Kingdom of Toledo: the Question of the Visigothic Cemeteries ", dans Völker, Reiche und Namen im frühen Mittelalter, 65. Geburtstag Prof. Dr. Jörg Jarnut, Paderborn/Bonn, sous presse.

- RIPOlL, ARCE, 2000 : Gisela Ripoll, Javier Arce Martínez, «The Transformation and End of Roman Villae in the West (Fourth-Seventh Centuries). Problems and Perspectives", dans Gian Pietro Brogiolo, Nancy Gauthier, Neil Christie éd., Towns and their Territories between Late Antiquity and the Early Middle Ages, Leyde/Boston, 2000, p. 63-114.

- RIPOLL, RIPOLL, 1988 : Gisela Ripoll, Odile Ripoll López, "Los conceptos de arqueología e historia del arte antiguo y medieval: apuntes historiográficos ", dans Espacio, Tiempo y Forma, 2/1, 1988, p. 411-426.

- RIPOLL, VELÁZQUEZ, 2000 : Gisela Ripoll, Isabel Velázquez Soriano, "Toletvm, la construcción de una vrbs regia ", dans Gisela Ripoll, Josep María Gurt Esparraguera éd., Sedes Regiae, ann. 400800, Barcelone, p. 521-578.

- RIPOLL, VelÁZQUeZ, 2008 : Gisela Ripoll, Isabel Velázquez Soriano, "Toletum vs. Recópolis: ¿Dos sedes para dos reyes? ", dans OLMO, 2008, p. 205-219.

- SCARROCCHIA, 1995 : Sandro Scarrocchia, Alois Riegl: teoria e prassi della conservazione dei monumenti : antologia di scritti, discorsi, rapporti 1898-1905, con una scelta di saggi critici, Bologne, 1995. -Schlunk, 1945 : Helmut Schlunk, "Observaciones en torno al problema de la miniatura visigoda ", dans Archivo Español de Arte, 117, 1945, p. 243-265. -SCHLunk, 1947 : Helmut Schlunk, "Arte visigodo " et "Arte asturiano", dans Blas Taracena Aguirre, Pedro Batlle Huguet, Helmut Schlunk éd., Ars Hispaniae, historia universal del arte hispánico, II, Arte romano, arte paleocristiano, arte visigodo, arte asturiano, Madrid, 1947, p. 225-416.

- SCHLUNK, HAUSCHILD, 1978 : Helmut Schlunk, Theodor Hauschild, Die Denkmäler der frühchristlichen und westgotischen Zeit, Mayence, 1978.

-Stylow, Gimeno, 2004 : Armin U. Stylow, Helena Gimeno Pascual, 
"Emil Hübner ", dans Zona arqueológica, 3, 2004, p. 333-340.

- Utrero, 2006 : María de los Ángeles Utrero Agudo, Iglesias tardoantiguas y altomedievales en la Península Ibérica. Análisis arqueológico y sistemas de abovedamiento, (Anejos de Archivo Español de Arqueología, 40), Madrid, 2006.

- Vigil-Escalera GUiRAdo, 2000 : Alfonso Vigil-Escalera Guirado, "Cabañas de época visigoda: evidencias arqueológicas del Sur de Madrid. Tipología, elementos de datación y discusión ", dans Archivo Español de Arqueología, 73, 2000, p. 223-252.

- VIGIL-ESCALERA GUIRAdO, 2005 : Alfonso Vigil-Escalera Guirado, " Nuevas perspectivas sobre la arqueología madrileña de época visigoda ", dans Actas de las Primeras Jornadas de Patrimonio Arqueológico en la Comunidad de Madrid, (colloque, Madrid, 2004), Madrid, 2005, p. 169-182.

- WARD-PERKINS, 2005 : Bryan WardPerkins, The Fall of Rome and the End of Civilization, Oxford/New York, 2005.

- Weddigen, 2003 : Tristan Weddigen, "Zur Funktionsgeschichte ", dans Tristan Weddigen, Sible de Blaa, Bram Kempers éd., Functions and Decorations: Art and Ritual at the Vatican Palace in the Middle Ages and the Renaissance, Vatican/ Turnhout, 2003, p. 9-25.

- Werner, 1942 : Joachim Werner, "Die Ausgrabung des westgotischen Gräberfeldes von Castiltierra (Prov. Segovia) in Jahre 1941 ", dans Forschungen und Fortschritte, 11/12, 1942, p. 108-109.

- WeRner, 1946 : Joachim Werner, "Las excavaciones del Seminario de Historia Primitiva del Hombre en 1941, en el cementerio visigodo de Castiltierra
(Segovia) ", dans Cuadernos de Historia Primitiva del Hombre, 1, 1946, p. 46-50.

-WicKHAM, 2005 : Chris Wickham, Framing the Early Middle Ages: Europe and the Mediterranean, 400-800, Oxford/ New York, 2005.

- WOLFRAM, 1990 : Herwig Wolfram, Histoire des Goths, Paris, 1990 [éd. orig. : Geschichte der Goten : von d. Anfängen bis zur Mitte d. 6. Jh, Munich, 1979].

- WOLFRAM, 1994 : Herwig Wolfram, "Origo et religio. Ethnic traditions and literature in early medieval texts ", dans Early Medieval Europe, 3/1, janvier 1994, p. 19-38.

- YARZA LUACES, 1985 : Joaquín Yarza Luaces, Arte asturiano, arte mozárabe, Cáceres, 1985.

- ZEISS, 1934 : Hans Zeiss, Die Grabfunde aus dem spanischen Westgotenreich, Berlin/ Leipzig, 1934. 